\title{
Synthesis of 6-Amino-3,4-Dihydroisoquinolinium Derivatives by Ring-Opening Reactions of Acridizinium lons
}

\author{
Hans-Jörg Deiseroth, ${ }^{a}$ Anton Granzhan, ${ }^{b}$ Heiko Ihmels, ${ }^{b, \star}$ Marc Schlosser, ${ }^{a}$ and \\ Maoqun Tian \\ Universität Siegen, Organische Chemie II ${ }^{b}$ and Anorganische Chemie I, ${ }^{a}$ Adolf-Reichwein-Str. 2, D-57068 \\ Siegen, Germany \\ ihmels@chemie.uni-siegen.de
}

\section{Supporting Information}

General Instruments and Materials

Synthetic details and characterization data for $\mathbf{2 a}-\mathbf{2 g}$ and $\mathbf{4}^{\prime}$

${ }^{1} \mathrm{H}-\mathrm{NMR}$ and ${ }^{13} \mathrm{C}-\mathrm{NMR}$ spectra of $\mathbf{2 a - 2 g}$ and $\mathbf{4}^{\prime}$ 
General Instrumentation and Materials. The melting points were measured with a melting point apparatus (Büchi 510K) and are uncorrected. Mass spectra (ESI in the positive-ion mode, source voltage $6 \mathrm{kV}$ ) were recorded with a Finnigan LCQ Deca instrument; only $\mathrm{m} / z$ values in the range of 100-2000 units were analyzed. NMR spectra were measured on a Bruker Avance $400\left({ }^{1} \mathrm{H}: 400 \mathrm{MHz},{ }^{13} \mathrm{C}: 100 \mathrm{MHz}\right)$ spectrometer at $20{ }^{\circ} \mathrm{C}$; chemical shifts are given in $\operatorname{ppm}(\delta)$ relative to TMS $(\delta=0.00 \mathrm{ppm})$. Unambiguous proton NMR assignments were established with the help of $\left\{{ }^{1} \mathrm{H},{ }^{1} \mathrm{H}\right\}$-COSY and, if necessary, HSQC and HMBC experiments. All coupling constants given in ${ }^{13} \mathrm{C}-\mathrm{NMR}$ spectroscopic data refer to ${ }^{13} \mathrm{C}-{ }^{19} \mathrm{~F}$ couplings. Elemental microanalyses of all new compounds were performed with a HEKAtech EuroEA combustion analyzer by Mr. H. Bodenstedt (Organische Chemie I, Universität Siegen). Absorption spectra were recorded on a Varian Cary 100 double-beam spectrophotometer; fluorescence emission spectra were recorded on a Varian Cary Eclipse fluorescence spectrometer. TLC analyses were performed on silica gel sheets (Macherey-Nagel Polygram Sil G/UV254), eluent: $\mathrm{CHCl}_{3} / \mathrm{MeOH} / \mathrm{AcOH}$ 90:10:1, v/v. All commercially available chemicals were reagent grade and used without further purification. 9-Aminoacridizinium and 9-fluoroacridizinium bromides were prepared according to the published procedures. ${ }^{1}$

\section{General procedure for the reaction of 9-fluoroacridizinium bromide with primary} amines. The corresponding amine $(6.60 \mathrm{mmol})$ was added to a suspension of 9-fluoroacridizinium bromide $(834 \mathrm{mg}, 3.00 \mathrm{mmol})$ in EtOH $(5 \mathrm{~mL})$ at room temperature. The reaction mixture became deep red and was heated under reflux for 6-24 h, until disappearance of the starting acridizinium derivative (TLC). After cooling to room temperature, the volatile components were removed in vacuo, and the residue was subjected to column chromatography $\left(\mathrm{SiO}_{2}\right.$, AcOEt-MeOH-AcOH 80:20:1). The blue-fluorescent fraction was collected, and the solution was filtered to remove traces of $\mathrm{SiO}_{2}$. The eluent was evaporated in vacuo. The corresponding perchlorates or hexafluorophosphates were prepared by addition of concentrated aqueous $\mathrm{NaClO}_{4}$ or $\mathrm{NH}_{4} \mathrm{PF}_{6}$ solution to the solution of the residue in aqueous $\mathrm{MeOH}$, followed by recrystallization of the precipitate from $\mathrm{MeOH}-\mathrm{AcOEt}$.

\footnotetext{
${ }^{1}$ (a) Ihmels, H.; Faulhaber, K.; Engels, B.; Lennartz, C. Chem. Eur. J. 2000, 6, 2854. (b) Bradsher, C. K.; Sherer, J. P.; Parham, J. H. J. Chem. Eng. Data 1965, 10, 180.
} 


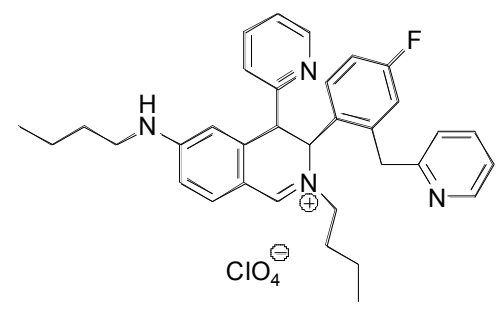

3-[4'-Fluoro-2'-(pyridin-2-ylmethyl)phenyl]-2-butyl-6-butylamino-4-pyridin-2-yl-3,4-di hydroisoquinolinium perchlorate (2a): yield 29\%, yellow needles; m.p. $178-180{ }^{\circ} \mathrm{C}$; ${ }^{1} \mathrm{H}-\mathrm{NMR}\left(400 \mathrm{MHz}, \mathrm{CD}_{3} \mathrm{COCD}_{3}\right): \delta=0.73\left(\mathrm{t}, 3 \mathrm{H},{ }^{3} J=7 \mathrm{~Hz}, 3 \mathrm{H}, \mathrm{CH}_{3}\right), 0.90\left(\mathrm{t}, 3 \mathrm{H},{ }^{3} J=7\right.$ $\left.\mathrm{Hz}, 3 \mathrm{H}, \mathrm{CH}_{3}\right), 0.96-1.16\left(\mathrm{~m}, 2 \mathrm{H}, \mathrm{CH}_{2}\right), 1.34-1.56\left(\mathrm{~m}, 4 \mathrm{H}, 2 \mathrm{CH}_{2}\right), 1.57-1.67\left(\mathrm{~m}, 2 \mathrm{H}, \mathrm{CH}_{2}\right)$, 3.29-3.36 (m, 2H, $\left.\mathrm{CH}_{2}\right), 3.50-3.58\left(\mathrm{~m}, 1 \mathrm{H}, \mathrm{CH}_{2}\right), 3.78-3.87\left(\mathrm{~m}, 1 \mathrm{H}, \mathrm{CH}_{2}\right), 4.37\left(\mathrm{~d},{ }^{2} \mathrm{~J}=15 \mathrm{~Hz}\right.$, $\left.1 \mathrm{H}, \mathrm{CH}_{2}\right), 4.79\left(\mathrm{~d},{ }^{2} J=15 \mathrm{~Hz}, 1 \mathrm{H}, \mathrm{CH}_{2}\right), 4.84(\mathrm{~s}, 1 \mathrm{H}, \mathrm{CH}), 6.48(\mathrm{~s}, 1 \mathrm{H}, \mathrm{CH}), 6.80-6.94(\mathrm{~m}$, $\left.3 \mathrm{H}, \mathrm{CH}_{\mathrm{ar}}\right), 7.05\left(\mathrm{~d},{ }^{3} \mathrm{~J}=8 \mathrm{~Hz}, 1 \mathrm{H}, \mathrm{CH}_{\mathrm{ar}}\right), 7.08-7.13\left(\mathrm{~m}, 1 \mathrm{H}, \mathrm{CH}_{\mathrm{ar}}\right), 7.21-7.25\left(\mathrm{~m}, 1 \mathrm{H}, \mathrm{CH}_{\mathrm{ar}}\right)$, 7.25-7.30 (m, 1H, $\left.\mathrm{CH}_{\mathrm{ar}}\right), 7.31-7.36\left(\mathrm{~m}, 1 \mathrm{H}, \mathrm{CH}_{\mathrm{ar}}\right), 7.54\left(\mathrm{~d},{ }^{3} \mathrm{~J}=8 \mathrm{~Hz}, 1 \mathrm{H}, \mathrm{CH}_{\mathrm{ar}}\right), 7.73-7.81$ $\left(\mathrm{m}, 2 \mathrm{H}, 2 \mathrm{CH}_{\mathrm{ar}}\right), 8.43\left(\mathrm{~m}, 1 \mathrm{H}, \mathrm{CH}_{\mathrm{ar}}\right), 8.62\left(\mathrm{~m}, 1 \mathrm{H}, \mathrm{CH}_{\mathrm{ar}}\right), 8.99\left(\mathrm{~s}, 1 \mathrm{H}, \mathrm{CH}_{\mathrm{ar}}\right) ;{ }^{13} \mathrm{C}-\mathrm{NMR}(100$ $\left.\mathrm{MHz}, \mathrm{CD}_{3} \mathrm{COCD}_{3}\right): \delta=13.8\left(\mathrm{CH}_{3}\right), 14.0\left(\mathrm{CH}_{3}\right), 19.9\left(\mathrm{CH}_{2}\right), 20.7\left(\mathrm{CH}_{2}\right), 30.7\left(\mathrm{CH}_{2}\right), 31.5$ $\left(\mathrm{CH}_{2}\right), 41.3\left(\mathrm{CH}_{2}\right), 43.4\left(\mathrm{CH}_{2}\right), 51.7(\mathrm{CH}), 58.0\left(\mathrm{CH}_{2}\right), 62.3(\mathrm{CH}), 113.3\left(\mathrm{C}_{\mathrm{q}}\right), 114.5(\mathrm{~d}, J=$ $\left.21 \mathrm{~Hz}, \mathrm{CH}_{\mathrm{ar}}\right), 119.6\left(\mathrm{~d}, J=22 \mathrm{~Hz}, \mathrm{CH}_{\mathrm{ar}}\right), 122.7\left(\mathrm{CH}_{\mathrm{ar}}\right), 122.8\left(\mathrm{CH}_{\mathrm{ar}}\right), 123.8\left(\mathrm{CH}_{\mathrm{ar}}\right), 124.3$ $\left(\mathrm{CH}_{\mathrm{ar}}\right), 128.3\left(\mathrm{~d}, J=9 \mathrm{~Hz}, \mathrm{CH}_{\mathrm{ar}}\right), 130.9(\mathrm{~d}, J=3 \mathrm{~Hz}, \mathrm{C} \mathrm{q}), 137.9\left(\mathrm{CH}_{\mathrm{ar}}\right), 138.3\left(\mathrm{CH}_{\mathrm{ar}}\right), 142.1$ $\left(\mathrm{d}, J=8 \mathrm{~Hz}, \mathrm{C}_{\mathrm{q}}\right), 150.2\left(\mathrm{CH}_{\mathrm{ar}}\right), 150.5\left(\mathrm{CH}_{\mathrm{ar}}\right), 158.7\left(\mathrm{C}_{\mathrm{q}}\right), 158.8\left(\mathrm{C}_{\mathrm{q}}\right), 160.6\left(\mathrm{C}_{\mathrm{q}}\right), 160.7\left(\mathrm{C}_{\mathrm{q}}\right)$, $163.2(\mathrm{CH}), 163.4\left(\mathrm{~d}, J=246 \mathrm{~Hz}, \mathrm{C}_{\mathrm{q}}\right)$; MS (ESI $\left.{ }^{+}\right): m / z(\%)=521$ (100) $\left[\mathrm{M}^{+}\right], 448(30)$ [M-C $\left.\mathrm{C}_{4} \mathrm{H}_{9} \mathrm{NH}^{+}\right]$; El. anal. calcd. (\%) for $\mathrm{C}_{34} \mathrm{H}_{38} \mathrm{ClFN}_{4} \mathrm{O}_{4}$ (621.1): C, 65.74; H, 6.17; N, 9.02; found: C, 65.89; H, 6.11; N, 8.93.

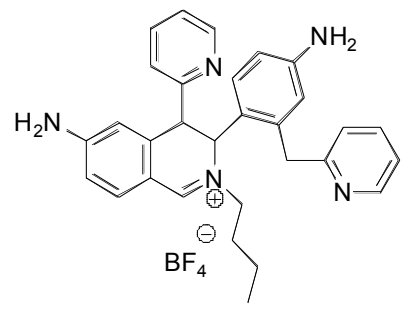

3-[4'-Amino-2'-(pyridin-2-ylmethyl)phenyl]-2-butyl-6-amino-4-pyridin-2-yl-3,4-dihydroisoquinolinium tetrafluoroborate (2b): Butylamine (146 mg, $2.00 \mathrm{mmol})$ was added to a suspension of 9-aminoacridizinium tetrafluoroborate $(200 \mathrm{mg}, 0.71 \mathrm{mmol})$ in $\mathrm{EtOH}(3 \mathrm{~mL})$ 
at room temperature. The reaction mixture was heated under reflux for $6 \mathrm{~h}$. After cooling to room temperature, dichloromethane $(50 \mathrm{~mL})$ was added to the reaction mixture. The organic phase was extracted with water $(30 \mathrm{~mL})$, brine $(30 \mathrm{~mL})$, and dried with sodium sulfate. After evaporating the solvent in vacuo, the remaining solid was recrystallized from dichloromethane to give the tetrafluoroborate salt (30 mg, $55 \mu \mathrm{mol}, 15 \%)$ as yellow prisms. ${ }^{1} \mathrm{H}-\mathrm{NMR}\left(400 \mathrm{MHz}, \mathrm{CD}_{3} \mathrm{OD}\right): \delta=0.71\left(\mathrm{t}, 3 \mathrm{H},{ }^{3} \mathrm{~J}=7 \mathrm{~Hz}, 3 \mathrm{H}, \mathrm{CH}_{3}\right), 0.85-1.03\left(\mathrm{~m}, 2 \mathrm{H}, \mathrm{CH}_{2}\right)$, 1.16-1.31 (m, 2H, $\left.\mathrm{CH}_{2}\right), 3.26-3.32\left(\mathrm{~m}, 1 \mathrm{H}, \mathrm{CH}_{2}\right), 3.51-3.58\left(\mathrm{~m}, 1 \mathrm{H}, \mathrm{CH}_{2}\right), 4.20\left(\mathrm{~d},{ }^{2} \mathrm{~J}=17 \mathrm{~Hz}\right.$, $\left.1 \mathrm{H}, \mathrm{CH}_{2}\right), 4.42(\mathrm{~s}, 1 \mathrm{H}, \mathrm{CH}), 4.54\left(\mathrm{~d},{ }^{2} J=17 \mathrm{~Hz}, 1 \mathrm{H}, \mathrm{CH}_{2}\right), 5.83(\mathrm{~s}, 1 \mathrm{H}, \mathrm{CH}), 6.46\left(\mathrm{dd},{ }^{3} J=8\right.$ $\left.\mathrm{Hz},{ }^{4} \mathrm{~J}=2 \mathrm{~Hz}, 1 \mathrm{H}, \mathrm{CH}_{\mathrm{ar}}\right), 6.55\left(\mathrm{~d},{ }^{4} \mathrm{~J}=2 \mathrm{~Hz}, 1 \mathrm{H}, \mathrm{CH}_{\mathrm{ar}}\right), 6.65\left(\mathrm{dd},{ }^{3} \mathrm{~J}=8 \mathrm{~Hz}, 1 \mathrm{H}, \mathrm{CH}_{\mathrm{ar}}\right), 6.68$ $\left(\mathrm{d},{ }^{4} \mathrm{~J}=2 \mathrm{~Hz}, 1 \mathrm{H}, \mathrm{CH}_{\mathrm{ar}}\right), 6.71\left(\mathrm{dd},{ }^{3} \mathrm{~J}=9 \mathrm{~Hz},{ }^{4} \mathrm{~J}=2 \mathrm{~Hz}, 1 \mathrm{H}, \mathrm{CH}_{\mathrm{ar}}\right), 6.84\left(\mathrm{~d},{ }^{3} \mathrm{~J}=8 \mathrm{~Hz}, 1 \mathrm{H}\right.$, $\mathrm{CH}_{\mathrm{ar}}$ ), 7.22-7.27 (m, 2H, $\left.\mathrm{CH}_{\mathrm{ar}}\right), 7.30\left(\mathrm{~d},{ }^{3} \mathrm{~J}=8 \mathrm{~Hz}, 1 \mathrm{H}, \mathrm{CH}_{\mathrm{ar}}\right), 7.61\left(\mathrm{~d},{ }^{3} \mathrm{~J}=9 \mathrm{~Hz}, 1 \mathrm{H}, \mathrm{CH}_{\mathrm{ar}}\right)$, 7.64-7.68 (m, $\left.1 \mathrm{H}, \mathrm{CH}_{\mathrm{ar}}\right), 7.73-7.77\left(\mathrm{~m}, 1 \mathrm{H}, \mathrm{CH}_{\mathrm{ar}}\right), 8.39\left(\mathrm{~d},{ }^{3} \mathrm{~J}=5 \mathrm{~Hz}, 1 \mathrm{H}, \mathrm{CH}_{\mathrm{ar}}\right), 8.56\left(\mathrm{~d},{ }^{3} J=\right.$ $\left.5 \mathrm{~Hz}, 1 \mathrm{H}, \mathrm{CH}_{\mathrm{ar}}\right), 8.64(\mathrm{~s}, 1 \mathrm{H}, \mathrm{CH}=\mathrm{N}) ;{ }^{13} \mathrm{C}-\mathrm{NMR}\left(100 \mathrm{MHz}, \mathrm{CD}_{3} \mathrm{OD}\right): \delta=13.9\left(\mathrm{CH}_{3}\right), 20.4$ $\left(\mathrm{CH}_{2}\right), 31.1\left(\mathrm{CH}_{2}\right), 42.2\left(\mathrm{CH}_{2}\right), 52.8(\mathrm{CH}), 58.2\left(\mathrm{CH}_{2}\right), 62.8(\mathrm{CH}), 113.8\left(\mathrm{C}_{\mathrm{q}}\right), 114.5\left(\mathrm{CH}_{\mathrm{ar}}\right)$, $114.9\left(\mathrm{CH}_{\mathrm{ar}}\right), 116.0\left(\mathrm{CH}_{\mathrm{ar}}\right), 119.6\left(\mathrm{CH}_{\mathrm{ar}}\right), 123.0\left(\mathrm{CH}_{\mathrm{ar}}\right), 123.1\left(\mathrm{C}_{\mathrm{q}}\right), 123.3\left(\mathrm{CH}_{\mathrm{ar}}\right), 124.0$ $\left(\mathrm{CH}_{\mathrm{ar}}\right), 124.9\left(\mathrm{CH}_{\mathrm{ar}}\right), 127.5\left(\mathrm{CH}_{\mathrm{ar}}\right), 138.1\left(\mathrm{CH}_{\mathrm{ar}}\right), 138.5\left(\mathrm{CH}_{\mathrm{ar}}\right), 138.8\left(\mathrm{CH}_{\mathrm{ar}}\right), 139.1\left(\mathrm{C}_{\mathrm{q}}\right)$, $140.0\left(\mathrm{C}_{\mathrm{q}}\right), 150.1\left(\mathrm{CH}_{\mathrm{ar}}\right), 150.3\left(\mathrm{C}_{\mathrm{q}}\right), 150.8\left(\mathrm{CH}_{\mathrm{ar}}\right), 160.8\left(\mathrm{C}_{\mathrm{q}}\right), 161.1\left(\mathrm{C}_{\mathrm{q}}\right), 161.6\left(\mathrm{C}_{\mathrm{q}}\right), 162.8$ $(\mathrm{CH}=\mathrm{N}) ; \mathrm{MS}\left(\mathrm{ESI}^{+}\right): m / z(\%)=463(100)\left[\mathrm{M}^{+}\right]$.

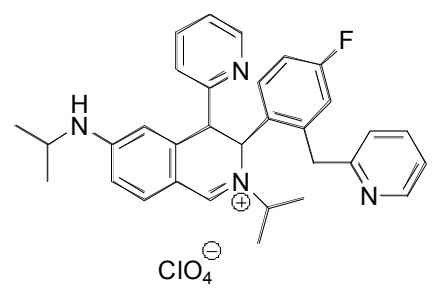

3-[4'-Fluoro-2'-(pyridin-2-ylmethyl)phenyl]-2-isopropyl-6-isopropylamino-4-pyridin-

2-yl-3,4-dihydroisoquinolinium perchlorate (2c): yield 81\%, yellow needles; m.p. 228-231

${ }^{\circ} \mathrm{C} ;{ }^{1} \mathrm{H}-\mathrm{NMR}\left[400 \mathrm{MHz},\left(\mathrm{CD}_{3}\right)_{2} \mathrm{SO}\right]: \delta=0.90\left(\mathrm{~d},{ }^{3} J=6 \mathrm{~Hz}, 3 \mathrm{H}, \mathrm{CH}_{3}\right), 1.03-1.26(\mathrm{~m}, 9 \mathrm{H}$, $\left.3 \mathrm{CH}_{3}\right), 3.66-3.81(\mathrm{~m}, 2 \mathrm{H}, \mathrm{CH}), 4.35\left(\mathrm{~d},{ }^{2} \mathrm{~J}=15 \mathrm{~Hz}, 1 \mathrm{H}, \mathrm{CH}_{2}\right), 4.63\left(\mathrm{~d},{ }^{2} J=15 \mathrm{~Hz}, 1 \mathrm{H}, \mathrm{CH}_{2}\right)$, $4.78(\mathrm{~s}, 1 \mathrm{H}, \mathrm{CH}), 6.15(\mathrm{~s}, 1 \mathrm{H}, \mathrm{CH}), 6.62$ (br s, $1 \mathrm{H}, \mathrm{NH}), 6.72\left(\mathrm{~d},{ }^{3} J=9 \mathrm{~Hz}, 1 \mathrm{H}, \mathrm{CH}_{\mathrm{ar}}\right)$, 6.88-7.03 (m, 3H, $\left.\mathrm{CH}_{\mathrm{ar}}\right), 7.24\left(\mathrm{dd},{ }^{3} \mathrm{~J}=8 \mathrm{~Hz},{ }^{3} \mathrm{~J}=5 \mathrm{~Hz}, 1 \mathrm{H}, \mathrm{CH}_{\mathrm{ar}}\right), 7.29-7.37\left(\mathrm{~m}, 2 \mathrm{H}, \mathrm{CH}_{\mathrm{ar}}\right)$, $7.53\left(\mathrm{~d},{ }^{3} \mathrm{~J}=8 \mathrm{~Hz}, 1 \mathrm{H}, \mathrm{CH}_{\mathrm{ar}}\right), 7.64-7.90\left(\mathrm{~m}, 4 \mathrm{H}, \mathrm{CH}_{\mathrm{ar}}\right), 8.40\left(\mathrm{~d},{ }^{3} \mathrm{~J}=5 \mathrm{~Hz}, 1 \mathrm{H}, \mathrm{CH}_{\mathrm{ar}}\right), 8.57$ (d, $\left.{ }^{3} J=4 \mathrm{~Hz}, 1 \mathrm{H}, \mathrm{CH}_{\mathrm{ar}}\right), 9.02(\mathrm{~s}, 1 \mathrm{H}, \mathrm{CH}) ;{ }^{13} \mathrm{C}-\mathrm{NMR}\left(100 \mathrm{MHz},\left(\mathrm{CD}_{3}\right)_{2} \mathrm{SO}\right): \delta=20.3\left(\mathrm{CH}_{3}\right)$, 
$20.7\left(\mathrm{CH}_{3}\right), 22.0\left(2 \mathrm{CH}_{3}\right), 40.1\left(\mathrm{CH}_{2}\right), 43.7(\mathrm{CH}), 50.0(\mathrm{CH}), 58.9(\mathrm{CH}), 60.4(\mathrm{CH}), 111.8$ $\left(\mathrm{C}_{\mathrm{q}}\right), 113.6\left(\mathrm{~d}, J=21 \mathrm{~Hz}, \mathrm{CH}_{\mathrm{ar}}\right), 118.8\left(\mathrm{~d}, J=23 \mathrm{~Hz}, \mathrm{CH}_{\mathrm{ar}}\right), 121.6\left(\mathrm{CH}_{\mathrm{ar}}\right), 122.0\left(\mathrm{CH}_{\mathrm{ar}}\right)$, $122.9\left(\mathrm{CH}_{\mathrm{ar}}\right), 123.6\left(\mathrm{CH}_{\mathrm{ar}}\right), 127.2\left(\mathrm{~d}, J=8 \mathrm{~Hz}, \mathrm{CH}_{\mathrm{ar}}\right), 130.5(\mathrm{~d}, J=3 \mathrm{~Hz}, \mathrm{C} \mathrm{q}), 137.3\left(\mathrm{CH}_{\mathrm{ar}}\right)$, $137.6\left(\mathrm{CH}_{\mathrm{ar}}\right), 140.6\left(\mathrm{~d}, J=8 \mathrm{~Hz}, \mathrm{C}_{\mathrm{q}}\right), 149.2\left(\mathrm{CH}_{\mathrm{ar}}\right), 149.5\left(\mathrm{CH}_{\mathrm{ar}}\right), 156.3\left(\mathrm{C}_{\mathrm{q}}\right), 159.3\left(\mathrm{C}_{\mathrm{q}}\right)$, $159.6\left(\mathrm{C}_{\mathrm{q}}\right), 159.7(\mathrm{~N}=\mathrm{CH}), 161.7\left(\mathrm{~d}, J=245 \mathrm{~Hz}, \mathrm{C}_{\mathrm{q}}\right)$; MS $\left(\mathrm{ESI}^{+}\right): m / z(\%)=493.6(30)\left[\mathrm{M}^{+}\right]$, $451.6(100)\left[\mathrm{M}^{+}-i-\mathrm{Pr}+\mathrm{H}\right]$; El. anal. calcd. (\%) for $\mathrm{C}_{32} \mathrm{H}_{34} \mathrm{ClFN}_{4} \mathrm{O}_{4}(593.1) \mathrm{C}, 64.80 ; \mathrm{H}, 5.78$; N, 9.45; found C, 64.67; H, 5.52; N, 9.29.

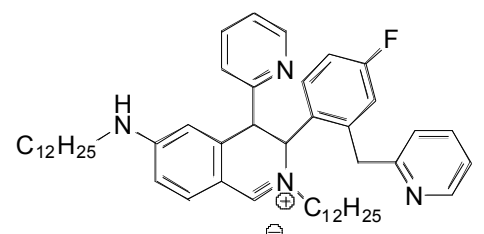
$\mathrm{CH}_{3} \mathrm{COO}^{\ominus}$

3-[4'-Fluoro-2'-(pyridin-2-ylmethyl)phenyl]-2-dodecyl-6-dodecylamino-4-pyridin-2yl-3,4-dihydroisoquinolinium acetate (2d): yield 55\%, brown oil; ${ }^{1} \mathrm{H}-\mathrm{NMR}(400 \mathrm{MHz}$, $\left.\mathrm{CD}_{3} \mathrm{OD}\right): \delta=0.88\left(\mathrm{t},{ }^{3} \mathrm{~J}=7 \mathrm{~Hz}, 3 \mathrm{H}, \mathrm{CH}_{3}\right), 0.89$ (t, $\left.{ }^{3} \mathrm{~J}=7 \mathrm{~Hz}, 3 \mathrm{H}, \mathrm{CH}_{3}\right), 0.93-1.69$ (m, 40H, $\mathrm{CH}_{2}$ ), 3.23 (br s, $2 \mathrm{H}, \mathrm{NCH}_{2}$ ), 3.29-3.37 (m, $\left.1 \mathrm{H}, \mathrm{NCH}_{2}\right), 3.55-3.66\left(\mathrm{~m}, 1 \mathrm{H}, \mathrm{NCH}_{2}\right), 4.38\left(\mathrm{~d},{ }^{2} J\right.$ $\left.=16,1 \mathrm{H}, \mathrm{CH}_{2}\right), 4.61($ br s, $1 \mathrm{H}, \mathrm{CH}), 4.79\left(\mathrm{~d},{ }^{2} \mathrm{~J}=16,1 \mathrm{H}, \mathrm{CH}_{2}\right), 6.10(\mathrm{~s}, 1 \mathrm{H}, \mathrm{CH}), 6.64(\mathrm{~s}, 1 \mathrm{H}$, $\left.\mathrm{CH}_{\mathrm{ar}}\right), 6.76\left(\mathrm{dd},{ }^{3} \mathrm{~J}=9 \mathrm{~Hz},{ }^{4} \mathrm{~J}=2 \mathrm{~Hz}, 1 \mathrm{H}, \mathrm{CH}_{\mathrm{ar}}\right), 6.82\left(\mathrm{~d},{ }^{3} \mathrm{~J}=8 \mathrm{~Hz}, 1 \mathrm{H}, \mathrm{CH}_{\mathrm{ar}}\right), 6.87-7.01(\mathrm{~m}$, $\left.2 \mathrm{H}, \mathrm{CH}_{\mathrm{ar}}\right), 7.12\left(\mathrm{dd},{ }^{3} J=10 \mathrm{~Hz},{ }^{4} J=3 \mathrm{~Hz} \mathrm{CH}_{\mathrm{ar}}\right), 7.26-7.28\left(\mathrm{~m}, 1 \mathrm{H}, \mathrm{CH}_{\mathrm{ar}}\right), 7.26-7.28(\mathrm{~m}, 1 \mathrm{H}$, $\left.\mathrm{CH}_{\mathrm{ar}}\right), 7.45\left(\mathrm{~d},{ }^{3} \mathrm{~J}=8 \mathrm{~Hz}, 1 \mathrm{H}, \mathrm{CH}_{\mathrm{ar}}\right), 7.67\left(\mathrm{dd},{ }^{3} \mathrm{~J}=8 \mathrm{~Hz},{ }^{3} \mathrm{~J}=8 \mathrm{~Hz}, 1 \mathrm{H}, \mathrm{CH}_{\mathrm{ar}}\right), 7.67\left(\mathrm{dd},{ }^{3} J=\right.$ $\left.8 \mathrm{~Hz},{ }^{3} J=8 \mathrm{~Hz}, 1 \mathrm{H}, \mathrm{CH}_{\mathrm{ar}}\right), 7.79-7.81\left(\mathrm{~m}, 1 \mathrm{H}, \mathrm{CH}_{\mathrm{ar}}\right), 8.42\left(\mathrm{~d},{ }^{3} J=4 \mathrm{~Hz}, 1 \mathrm{H}, \mathrm{CH}_{\mathrm{ar}}\right), 8.60\left(\mathrm{~d},{ }^{3} J\right.$ $\left.=4 \mathrm{~Hz}, 1 \mathrm{H}, \mathrm{CH}_{\mathrm{ar}}\right), 8.72(\mathrm{~s}, 1 \mathrm{H}, \mathrm{N}=\mathrm{CH}) ;{ }^{13} \mathrm{C}-\mathrm{NMR}\left(100 \mathrm{MHz}, \mathrm{CD}_{3} \mathrm{OD}\right): \delta=14.6\left(\mathrm{CH}_{3}\right), 23.9$ $\left(\mathrm{CH}_{3}\right), 27.1\left(\mathrm{CH}_{2}\right), 28.2\left(\mathrm{CH}_{2}\right), 29.3\left(\mathrm{CH}_{2}\right), 30.0\left(\mathrm{CH}_{2}\right), 30.2\left(\mathrm{CH}_{2}\right), 30.5\left(\mathrm{CH}_{2}\right), 30.5\left(\mathrm{CH}_{2}\right)$, $30.5\left(\mathrm{CH}_{2}\right), 30.6\left(\mathrm{CH}_{2}\right), 30.6\left(\mathrm{CH}_{2}\right), 30.7\left(\mathrm{CH}_{2}\right), 30.7\left(\mathrm{CH}_{2}\right), 30.7\left(\mathrm{CH}_{2}\right), 33.1\left(\mathrm{CH}_{2}\right), 41.6$ $\left(\mathrm{CH}_{2}\right), 44.2\left(\mathrm{CH}_{2}\right), 52.5(\mathrm{CH}), 58.6\left(\mathrm{CH}_{2}\right), 62.7(\mathrm{CH}), 113.7\left(\mathrm{C}_{\mathrm{q}}\right), 115.3\left(\mathrm{~d}, J=22 \mathrm{~Hz}, \mathrm{CH}_{\mathrm{ar}}\right)$, $120.3\left(\mathrm{~d}, J=23 \mathrm{~Hz}, \mathrm{CH}_{\mathrm{ar}}\right), 123.2\left(\mathrm{CH}_{\mathrm{ar}}\right), 123.6\left(\mathrm{CH}_{\mathrm{ar}}\right), 124.3\left(\mathrm{CH}_{\mathrm{ar}}\right), 125.3\left(\mathrm{CH}_{\mathrm{ar}}\right), 128.8(\mathrm{~d}$, $\left.J=8 \mathrm{~Hz}, \mathrm{CH}_{\mathrm{ar}}\right), 131.2\left(\mathrm{~d}, J=3 \mathrm{~Hz}, \mathrm{C}_{\mathrm{q}}\right), 138.8\left(\mathrm{CH}_{\mathrm{ar}}\right), 139.0\left(\mathrm{CH}_{\mathrm{ar}}\right), 141.7(\mathrm{~d}, J=7 \mathrm{~Hz}, \mathrm{C})$, $150.5\left(\mathrm{CH}_{\mathrm{ar}}\right), 151.0\left(\mathrm{CH}_{\mathrm{ar}}\right), 159.8\left(\mathrm{CH}_{\mathrm{ar}}\right), 160,9\left(\mathrm{CH}_{\mathrm{ar}}\right), 160.9\left(\mathrm{CH}_{\mathrm{ar}}\right), 163.1(\mathrm{~N}=\mathrm{CH}), 164.4$ $\left(\mathrm{d}, J=247 \mathrm{~Hz}, \mathrm{C}_{\mathrm{q}}\right) ; \mathrm{MS}\left(\mathrm{ESI}^{+}\right): m / z(\%)=746(100)\left[\mathrm{M}^{+}\right], 561(20)\left[\mathrm{M}^{+}-\mathrm{HN}-\right.$ dodecyl $]$. 


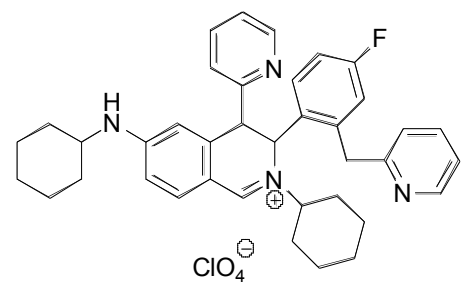

3-[4'-Fluoro-2'-(pyridin-2-ylmethyl)phenyl]-2-cyclohexcyl-6-cyclohexylamino-4-pyridin-2-yl-3,4-dihydroisoquinolinium perchlorate (2e): yield 70\%, yellow needles; m.p. 188-190 ${ }^{\circ} \mathrm{C}$; ${ }^{1} \mathrm{H}-\mathrm{NMR}\left(400 \mathrm{MHz}, \mathrm{CD}_{3} \mathrm{OD}\right): \delta=0.84-2.00\left(\mathrm{~m}, 20 \mathrm{H}, \mathrm{CH}_{2}\right.$ ), 3.23 (br s, $1 \mathrm{H}$, $\mathrm{NCH}), 3.45$ (br s, $1 \mathrm{H}, \mathrm{NCH}), 4.38\left(\mathrm{~d},{ }^{2} J=15,1 \mathrm{H}, \mathrm{CH}_{2}\right), 4.60$ (br s, $\left.1 \mathrm{H}, \mathrm{CH}\right), 4.79\left(\mathrm{~d},{ }^{2} J=15\right.$ $\left.\mathrm{Hz}, 1 \mathrm{H}, \mathrm{CH}_{2}\right), 6.04(\mathrm{~s}, 1 \mathrm{H}, \mathrm{CH}), 6.59\left(\mathrm{~s}, 1 \mathrm{H}, \mathrm{CH}_{\mathrm{ar}}\right), 6.74\left(\mathrm{dd},{ }^{3} J=9 \mathrm{~Hz},{ }^{4} J=2 \mathrm{~Hz}, 1 \mathrm{H}, \mathrm{CH}_{\mathrm{ar}}\right)$, $6.84\left(\mathrm{~d},{ }^{3} \mathrm{~J}=8 \mathrm{~Hz}, 1 \mathrm{H}, \mathrm{CH}_{\mathrm{ar}}\right), 6.85-6.98\left(\mathrm{~m}, 2 \mathrm{H}, \mathrm{CH}_{\mathrm{ar}}\right), 7.15\left(\mathrm{dd},{ }^{3} \mathrm{~J}=10 \mathrm{~Hz},{ }^{4} \mathrm{~J}=3 \mathrm{~Hz}, 1 \mathrm{H}\right.$, $\left.\mathrm{CH}_{\mathrm{ar}}\right), 7.27-7.29\left(\mathrm{~m}, 1 \mathrm{H}, \mathrm{CH}_{\mathrm{ar}}\right), 7.29-7.31\left(\mathrm{~m}, 1 \mathrm{H}, \mathrm{CH}_{\mathrm{ar}}\right), 7.54\left(\mathrm{~d},{ }^{3} J=8 \mathrm{~Hz}, 1 \mathrm{H}, \mathrm{CH}_{\mathrm{ar}}\right), 7.68$ $\left(\mathrm{dd},{ }^{3} \mathrm{~J}=8 \mathrm{~Hz},{ }^{3} \mathrm{~J}=8 \mathrm{~Hz}, 1 \mathrm{H}, \mathrm{CH}_{\mathrm{ar}}\right), 7.68\left(\mathrm{dd},{ }^{3} \mathrm{~J}=8 \mathrm{~Hz},{ }^{3} \mathrm{~J}=8 \mathrm{~Hz}, 1 \mathrm{H}, \mathrm{CH}_{\mathrm{ar}}\right), 7.82-7.84(\mathrm{~m}$, $\left.1 \mathrm{H}, \mathrm{CH}_{\mathrm{ar}}\right), 8.46\left(\mathrm{~d},{ }^{3} \mathrm{~J}=4 \mathrm{~Hz}, 1 \mathrm{H}, \mathrm{CH}_{\mathrm{ar}}\right), 8.64\left(\mathrm{~d},{ }^{3} \mathrm{~J}=5 \mathrm{~Hz}, 1 \mathrm{H}, \mathrm{CH}_{\mathrm{ar}}\right), 8.75(\mathrm{~s}, 1 \mathrm{H}, \mathrm{N}=\mathrm{CH})$; ${ }^{13} \mathrm{C}-\mathrm{NMR}\left(100 \mathrm{MHz}, \mathrm{CD}_{3} \mathrm{OD}\right): \delta=25.7\left(\mathrm{CH}_{2}\right), 25.8\left(2 \mathrm{CH}_{2}\right), 26.2\left(\mathrm{CH}_{2}\right), 26.3\left(\mathrm{CH}_{2}\right), 26.5$ $\left(\mathrm{CH}_{2}\right), 32.5\left(\mathrm{CH}_{2}\right), 33.1\left(\mathrm{CH}_{2}\right), 33.5\left(2 \mathrm{CH}_{2}\right), 41.2\left(\mathrm{CH}_{2}\right), 52.6(\mathrm{NCH}), 52.8(\mathrm{NCH}), 63.0$ $(\mathrm{CH}), 68.6(\mathrm{NCH}), 113.8\left(\mathrm{C}_{\mathrm{q}}\right), 115.1\left(\mathrm{~d}, J=21 \mathrm{~Hz}, \mathrm{CH}_{\mathrm{ar}}\right), 120.4\left(\mathrm{~d}, J=21 \mathrm{~Hz}, \mathrm{CH}_{\mathrm{ar}}\right), 123.0$ $\left(\mathrm{CH}_{\mathrm{ar}}\right), 124.0\left(\mathrm{CH}_{\mathrm{ar}}\right), 124.2\left(\mathrm{CH}_{\mathrm{ar}}\right), 125.8\left(\mathrm{CH}_{\mathrm{ar}}\right), 128.9\left(\mathrm{~d}, J=9 \mathrm{~Hz}, \mathrm{CH}_{\mathrm{ar}}\right), 131.3(\mathrm{~d}, J=3 \mathrm{~Hz}$, $\left.\mathrm{C}_{\mathrm{q}}\right), 138.7\left(\mathrm{CH}_{\mathrm{ar}}\right), 140.0\left(\mathrm{CH}_{\mathrm{ar}}\right), 140.7\left(\mathrm{~d}, J=7 \mathrm{~Hz}, \mathrm{C}_{\mathrm{q}}\right), 149.8\left(\mathrm{CH}_{\mathrm{ar}}\right), 150.8\left(\mathrm{CH}_{\mathrm{ar}}\right), 158.5$ $\left(\mathrm{C}_{\mathrm{q}}\right), 160.1\left(\mathrm{C}_{\mathrm{q}}\right), 160.6\left(\mathrm{C}_{\mathrm{q}}\right), 160.8(\mathrm{~N}=\mathrm{CH}), 164.2\left(\mathrm{~d}, J=248 \mathrm{~Hz}, \mathrm{C}_{\mathrm{q}}\right)$; MS $\left(\mathrm{ESI}^{+}\right): m / z(\%)=$ 574 (100) $\left[\mathrm{M}^{+}\right], 475$ (24) $\left[\mathrm{M}^{+}-\mathrm{NH}_{2} \mathrm{C}_{6} \mathrm{H}_{11}\right]$; El. Anal. calcd. (\%) for $\mathrm{C}_{38} \mathrm{H}_{42} \mathrm{ClFN}_{4} \mathrm{O}_{4}(673.22)$ : C, 67.79; H, 6.29; N, 8.32; found: C, 67.74; H, 6.05; N, 8.27.

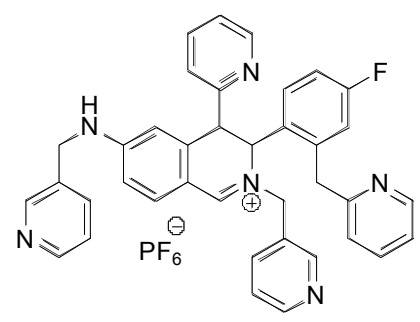

3-[4'-Fluoro-2'-(pyridin-2-ylmethyl)phenyl]-2-(pyridin-3-yl)methyl-6-(pyridin-3-yl)methylamino-4-pyridin-2-yl-3,4-dihydroisoquinolinium (2f): isolated as hydrogen hexafluorophosphate; yield 20\%, yellow plates; m.p. 186-188 ${ }^{\circ} \mathrm{C} ;{ }^{1} \mathrm{H}-\mathrm{NMR}(400 \mathrm{MHz}$, $\left.\mathrm{CD}_{3} \mathrm{COCD}_{3}\right): \delta=4.43\left(\mathrm{~d}, 1 \mathrm{H},{ }^{2} J=15 \mathrm{~Hz}, \mathrm{CH}_{2}\right), 4.95\left(\mathrm{~d}, 1 \mathrm{H},{ }^{2} J=15 \mathrm{~Hz}, \mathrm{CH}_{2}\right), 4.81-4.92(\mathrm{~m}$, 
$\left.3 \mathrm{H}, \mathrm{CH}_{2}, \mathrm{CH}\right), 4.95\left(\mathrm{~d}, 1 \mathrm{H},{ }^{2} J=15 \mathrm{~Hz}, \mathrm{CH}_{2}\right), 5.34\left(\mathrm{~d}, 1 \mathrm{H},{ }^{2} J=15 \mathrm{~Hz}, \mathrm{CH}_{2}\right), 6.29(\mathrm{~s}, 1 \mathrm{H}, \mathrm{CH})$, 6.59 (br s, $1 \mathrm{H}, \mathrm{NH}), 6.85-7.06\left(\mathrm{~m}, 4 \mathrm{H}, \mathrm{CH}_{\mathrm{ar}}\right), 7.18-7.30\left(\mathrm{~m}, 3 \mathrm{H}, \mathrm{CH}_{\mathrm{ar}}\right), 7.36-7.45(\mathrm{~m}, 2 \mathrm{H}$, $\left.\mathrm{CH}_{\mathrm{ar}}\right), 7.59\left(\mathrm{~d}, 1 \mathrm{H},{ }^{3} \mathrm{~J}=8 \mathrm{~Hz}, \mathrm{CH}_{\mathrm{ar}}\right), 7.62-7.69\left(\mathrm{~m}, 1 \mathrm{H}, \mathrm{CH}_{\mathrm{ar}}\right), 7.77-7.98\left(\mathrm{~m}, 5 \mathrm{H}, \mathrm{CH}_{\mathrm{ar}}\right), 8.31(\mathrm{~d}$, $\left.1 \mathrm{H},{ }^{3} J=5 \mathrm{~Hz}, \mathrm{CH}_{\mathrm{ar}}\right), 8.43\left(\mathrm{~d}, 1 \mathrm{H},{ }^{3} J=8 \mathrm{~Hz}, \mathrm{CH}_{\mathrm{ar}}\right), 8.50-8.73\left(\mathrm{~m}, 3 \mathrm{H}, \mathrm{CH}_{\mathrm{ar}}\right), 8.86(\mathrm{~s}, 1 \mathrm{H}$, $\left.\mathrm{CH}_{\mathrm{ar}}\right), 8.94\left(\mathrm{~s}, 1 \mathrm{H}, \mathrm{CH}_{\mathrm{ar}}\right), 9.35(\mathrm{~s}, 1 \mathrm{H}, \mathrm{N}=\mathrm{CH}) ;{ }^{13} \mathrm{C}-\mathrm{NMR}\left(100 \mathrm{MHz}, \mathrm{CD}_{3} \mathrm{COCD}_{3}\right): \delta=40.1$ $\left(\mathrm{CH}_{2}\right), 44.5\left(\mathrm{CH}_{2}\right), 51.5(\mathrm{CH}), 59.0\left(\mathrm{CH}_{2}\right), 62.8(\mathrm{CH}), 114.3\left(\mathrm{C}_{\mathrm{q}}\right), 115.1\left(\mathrm{~d}, J=21 \mathrm{~Hz}, \mathrm{CH}_{\mathrm{ar}}\right)$, $119.8\left(\mathrm{~d}, J=22 \mathrm{~Hz}, \mathrm{CH}_{\mathrm{ar}}\right), 122.7\left(\mathrm{CH}_{\mathrm{ar}}\right), 123.7\left(\mathrm{CH}_{\mathrm{ar}}\right), 123.9\left(\mathrm{CH}_{\mathrm{ar}}\right), 125.2\left(\mathrm{CH}_{\mathrm{ar}}\right), 125.4\left(\mathrm{C}_{\mathrm{q}}\right)$, $127.2\left(\mathrm{CH}_{\mathrm{ar}}\right), 128.7$ (d, $\left.J=8 \mathrm{~Hz}, \mathrm{CH}_{\mathrm{ar}}\right), 129.9\left(\mathrm{~d}, J=3 \mathrm{~Hz}, \mathrm{C}_{\mathrm{q}}\right), 130.5\left(\mathrm{C}_{\mathrm{q}}\right), 137.9\left(\mathrm{C}_{\mathrm{q}}\right), 138.4$ $\left(\mathrm{CH}_{\mathrm{ar}}\right), 139.4\left(\mathrm{CH}_{\mathrm{ar}}\right), 140.2\left(\mathrm{CH}_{\mathrm{ar}}\right), 141.1\left(\mathrm{~d}, J=8 \mathrm{~Hz}, \mathrm{C}_{\mathrm{q}}\right), 143.2\left(\mathrm{CH}_{\mathrm{ar}}\right), 144.8\left(\mathrm{CH}_{\mathrm{ar}}\right), 144.8$ $\left(\mathrm{CH}_{\mathrm{ar}}\right), 149.0\left(\mathrm{CH}_{\mathrm{ar}}\right), 149.5\left(\mathrm{CH}_{\mathrm{ar}}\right), 150.4\left(\mathrm{CH}_{\mathrm{ar}}\right), 158.6\left(\mathrm{C}_{\mathrm{q}}\right) 158.7\left(\mathrm{C}_{\mathrm{q}}\right), 159.3\left(\mathrm{C}_{\mathrm{q}}\right), 159.8\left(\mathrm{C}_{\mathrm{q}}\right)$, $163.7\left(\mathrm{~d}, J=246 \mathrm{~Hz}, \mathrm{C}_{\mathrm{q}}\right) 165.0(\mathrm{CH}) ; \mathrm{MS}\left(\mathrm{ESI}^{+}\right): m / z(\%) 591(100)\left[\mathrm{M}^{+}\right], 483(5)\left[\mathrm{M}^{+}-\right.$ $\mathrm{NH}_{2} \mathrm{C}_{6} \mathrm{H}_{6} \mathrm{~N}$ ]; El. Anal. calcd. (\%) for $\mathrm{C}_{38} \mathrm{H}_{33} \mathrm{~F}_{13} \mathrm{~N}_{6} \mathrm{P}_{2}$ (868.61): C, 51.71; H, 3.77; N, 9.52; found: C, 51.52; H, 3.57; N, 9.20.

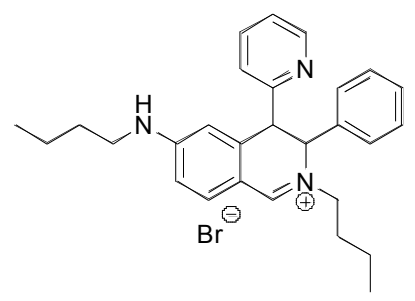

\section{3-Phenyl-2-butyl-6-butylamino-4-pyridin-2-yl-3,4-dihydroisoquinolinium bromide (2g):}

Benzaldehyde (768 mg, $7.25 \mathrm{mmol})$ and butylamine $(318 \mathrm{mg}, 4.35 \mathrm{mmol})$ were added to a suspension of 9-fluoroacridizinium bromide (400 mg, $1.45 \mathrm{mmol})$ in EtOH $(10 \mathrm{~mL})$ at room temperature. The reaction mixture was heated under reflux for $5 \mathrm{~h}$. After cooling to room temperature, the volatile components were removed in vacuo, and the residue was subjected to column chromatography $\left(\mathrm{SiO}_{2}, \mathrm{CHCl}_{3} / \mathrm{MeOH} 10: 1, R_{\mathrm{f}}=0.4\right)$. The blue fluorescent fraction was collected, and the solution was filtered to remove traces of $\mathrm{SiO}_{2}$. The solvent was removed in vacuo to yield $395 \mathrm{mg}(55 \%)$ of the product as yellow oil. ${ }^{1} \mathrm{H}-\mathrm{NMR}$ (400 $\left.\mathrm{MHz}, \mathrm{CD}_{3} \mathrm{OD}\right): \delta=0.77\left(\mathrm{t},{ }^{3} J=7 \mathrm{~Hz}, 3 \mathrm{H}, \mathrm{CH}_{3}\right), 0.92\left(\mathrm{t},{ }^{3} J=7 \mathrm{~Hz}, 3 \mathrm{H}, \mathrm{CH}_{3}\right), 0.98-1.06(\mathrm{~m}$, $\left.1 \mathrm{H}, \mathrm{CH}_{2}\right), 1.07-1.15\left(\mathrm{~m}, 1 \mathrm{H}, \mathrm{CH}_{2}\right), 1.35-1.42\left(\mathrm{~m}, 2 \mathrm{H}, \mathrm{CH}_{2}\right), 1.42-1.48\left(\mathrm{~m}, 1 \mathrm{H}, \mathrm{CH}_{2}\right)$, 1.48-1.54 (m, $\left.1 \mathrm{H}, \mathrm{CH}_{2}\right), 1.55-1.64\left(\mathrm{~m}, 2 \mathrm{H}, \mathrm{CH}_{2}\right), 3.22$ (br s, $\left.2 \mathrm{H}, \mathrm{NCH}_{2}\right), 3.51-3.57(\mathrm{~m}, 1 \mathrm{H}$, $\left.\mathrm{NCH}_{2}\right), 3.76-3.83\left(\mathrm{~m}, 1 \mathrm{H}, \mathrm{NCH}_{2}\right), 4.64$ (br s, $\left.1 \mathrm{H}, \mathrm{CH}\right), 5.67$ (s, 1H, CH), $6.60\left(\mathrm{~s}, 1 \mathrm{H}, \mathrm{CH}_{\mathrm{ar}}\right.$ ), $6.74\left(\mathrm{~d},{ }^{3} J=9 \mathrm{~Hz}, 1 \mathrm{H}, \mathrm{CH}_{\mathrm{ar}}\right), 7.00\left(\mathrm{~d},{ }^{3} J=8 \mathrm{~Hz}, 1 \mathrm{H}, \mathrm{CH}_{\mathrm{ar}}\right), 7.31-7.40\left(\mathrm{~m}, 6 \mathrm{H}, \mathrm{CH}_{\mathrm{ar}}\right)$, 
7.65-7.81 (m, 2H, $\left.\mathrm{CH}_{\mathrm{ar}}\right), 7.91\left(\mathrm{~s}, 1 \mathrm{H}, \mathrm{CH}_{\mathrm{ar}}\right), 8.62\left(\mathrm{~d},{ }^{3} \mathrm{~J}=5 \mathrm{~Hz}, 1 \mathrm{H}, \mathrm{CH}_{\mathrm{ar}}\right), 8.80(\mathrm{~s}, 1 \mathrm{H}$, $\mathrm{CH}=\mathrm{N}) ;{ }^{13} \mathrm{C}-\mathrm{NMR}\left(100 \mathrm{MHz}, \mathrm{CD}_{3} \mathrm{OD}\right): \delta=13.8\left(\mathrm{CH}_{3}\right), 14.1\left(\mathrm{CH}_{3}\right), 20.3\left(\mathrm{CH}_{2}\right), 21.1\left(\mathrm{CH}_{2}\right)$, $31.1\left(\mathrm{CH}_{2}\right), 31.9\left(\mathrm{CH}_{2}\right), 43.7\left(\mathrm{CH}_{2}\right), 53.5(\mathrm{CH}), 58.5\left(\mathrm{NCH}_{2}\right), 66.6(\mathrm{CH}), 113.9\left(\mathrm{C}_{\mathrm{q}}\right), 123.6$ $\left(\mathrm{CH}_{\mathrm{ar}}\right), 124.3\left(\mathrm{CH}_{\mathrm{ar}}\right), 127.5\left(\mathrm{CH}_{\mathrm{ar}}\right), 130.1\left(\mathrm{CH}_{\mathrm{ar}}\right), 130.4\left(\mathrm{CH}_{\mathrm{ar}}\right), 136.7\left(\mathrm{C}_{\mathrm{q}}\right), 138.9\left(\mathrm{CH}_{\mathrm{ar}}\right)$, $150.7\left(\mathrm{CH}_{\mathrm{ar}}\right), 159.5\left(\mathrm{C}_{\mathrm{q}}\right), 160.5\left(\mathrm{C}_{\mathrm{q}}\right), 162.5(\mathrm{CH}=\mathrm{N})$; MS $\left(\mathrm{ESI}^{+}\right): m / z(\%) 412(100)\left[\mathrm{M}^{+}\right]$; El. Anal. calcd. (\%) for $\mathrm{C}_{28} \mathrm{H}_{34} \mathrm{BrN}_{3} \times 1 / 2 \mathrm{H}_{2} \mathrm{O}$ (408.72): C, 67.06; H, 7.03; N, 8.38; found: C, $66.85 ; \mathrm{H}, 6.91 ; \mathrm{N}, 8.25$.

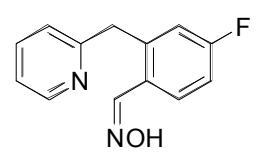

2-(Pyridin-2-ylmethyl)-4-fluorobenzaldoxime (4'): A solution of hydroxylamine hydrochloride (400 mg, $5.76 \mathrm{mmol}$ ), neutralized by addition of excess sodium bicarbonate, was rapidly added to a stirred solution containing 9-fluoroacridizinium bromide (834 $\mathrm{mg}$, $3.00 \mathrm{mmol}$ ) in $15 \mathrm{ml}$ of water. A brown solid precipitated immediately, and the solution was stirred for $40 \mathrm{~min}$ at r.t. The precipitate was collected and recrystallized from ethanol yielding $573 \mathrm{mg}$ (83\%) of the product as colorless plates. m.p. $95-98{ }^{\circ} \mathrm{C} ;{ }^{1} \mathrm{H}-\mathrm{NMR}(400 \mathrm{MHz}$, $\left.\mathrm{CD}_{3} \mathrm{OD}\right): \delta=4.36\left(\mathrm{~s}, 2 \mathrm{H}, \mathrm{CH}_{2}\right), 6.95-7.08\left(\mathrm{~m}, 2 \mathrm{H}, \mathrm{CH}_{\mathrm{ar}}\right), 7.19\left(\mathrm{~d}, 1 \mathrm{H},{ }^{3} \mathrm{~J}=8 \mathrm{~Hz}, \mathrm{CH}_{\mathrm{ar}}\right), 7.28$ $\left(\mathrm{dd}, 1 \mathrm{H},{ }^{3} J=7 \mathrm{~Hz},{ }^{3} \mathrm{~J}=5 \mathrm{~Hz}\right), 7.75\left(\mathrm{~m}, 2 \mathrm{H}, \mathrm{CH}_{\mathrm{ar}}\right), 8.33(\mathrm{~s}, 1 \mathrm{H}, \mathrm{HC}=\mathrm{N}), 8.49\left(\mathrm{~d}, 1 \mathrm{H},{ }^{3} J=5\right.$ $\left.\mathrm{Hz}, \mathrm{CH}_{\mathrm{ar}}\right) ;{ }^{13} \mathrm{C}-\mathrm{NMR}\left(100 \mathrm{MHz}, \mathrm{CD}_{3} \mathrm{OD}\right): \delta=41.9\left(\mathrm{~d}, J=1 \mathrm{~Hz}, \mathrm{CH}_{2}\right), 115.0(\mathrm{~d}, J=21 \mathrm{~Hz}$, $\left.\mathrm{CH}_{\mathrm{ar}}\right), 118.5\left(\mathrm{~d}, J=23 \mathrm{~Hz}, \mathrm{CH}_{\mathrm{ar}}\right), 123.2\left(\mathrm{CH}_{\mathrm{ar}}\right), 124.9\left(\mathrm{CH}_{\mathrm{ar}}\right), 129.3\left(\mathrm{~d}, J=3 \mathrm{~Hz}, \mathrm{C}_{\mathrm{q}}\right), 130.5$ $\left(\mathrm{d}, J=8 \mathrm{~Hz}, \mathrm{CH}_{\mathrm{ar}}\right), 138.9\left(\mathrm{CH}_{\mathrm{ar}}\right), 141.3\left(\mathrm{~d}, J=8 \mathrm{~Hz}, \mathrm{C}_{\mathrm{q}}\right), 147.9(\mathrm{~N}=\mathrm{CH}), 149.9\left(\mathrm{CH}_{\mathrm{ar}}\right) 160.9$ $\left(\mathrm{C}_{\mathrm{q}}\right), 164.6\left(\mathrm{~d}, J=249.0 \mathrm{~Hz}, \mathrm{C}_{\mathrm{q}}\right)$; El. anal. calcd. (\%) for $\mathrm{C}_{13} \mathrm{H}_{11} \mathrm{FN}_{2} \mathrm{O}$ (230.2) C: 67.82, $\mathrm{H}$ : 4.82, N: 12.17; found C: 67.62, H: 4.61, N: 11.96 . 


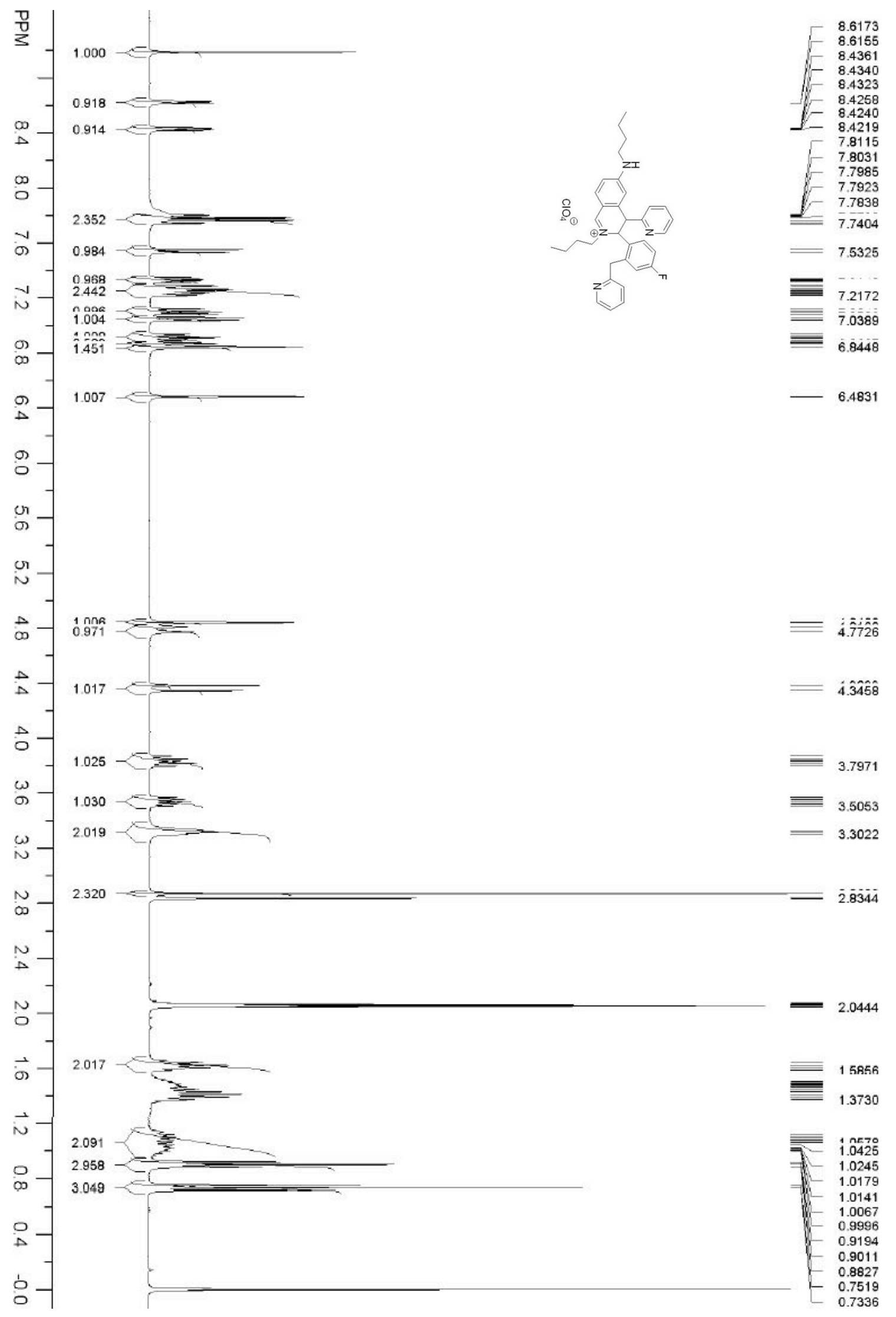




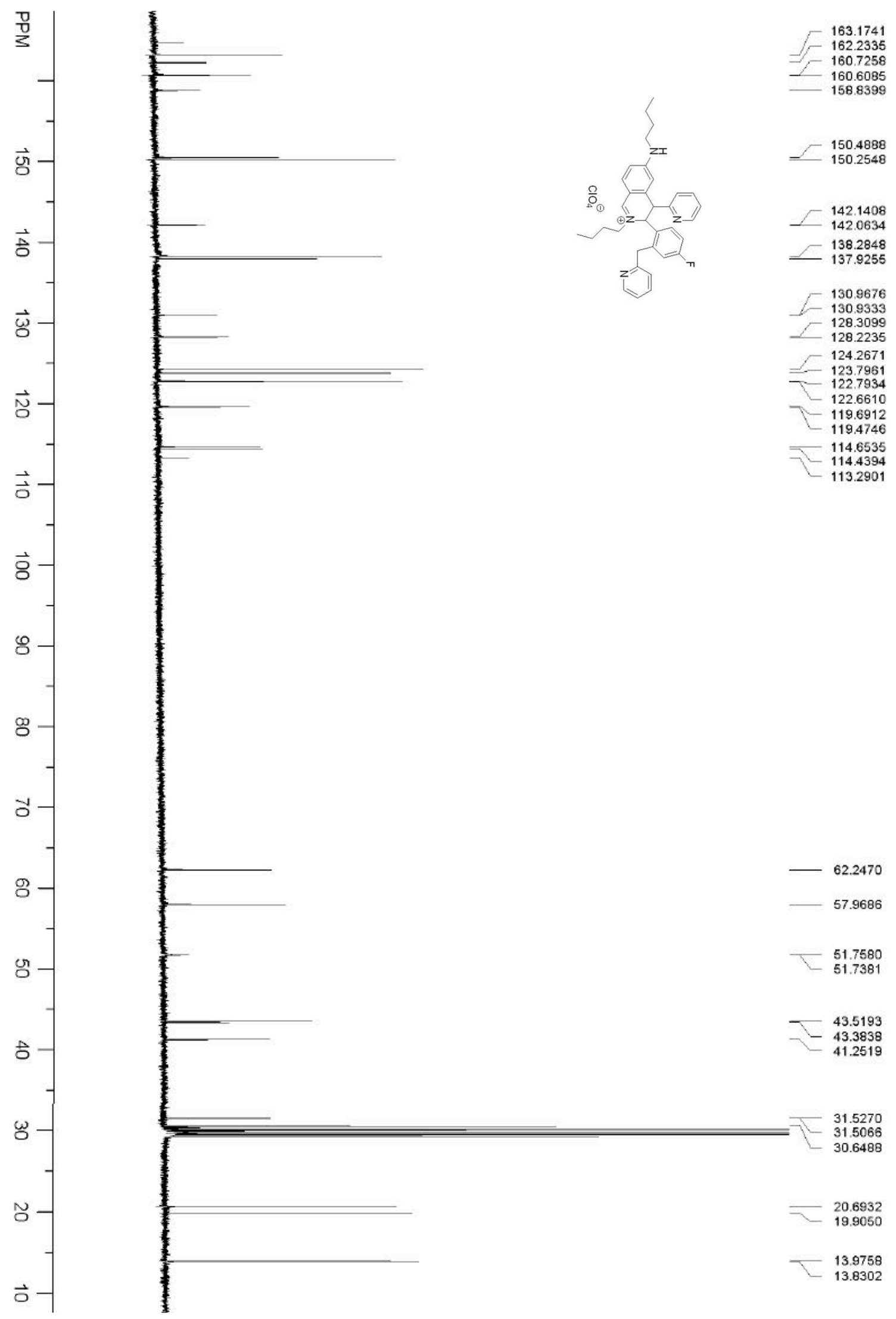




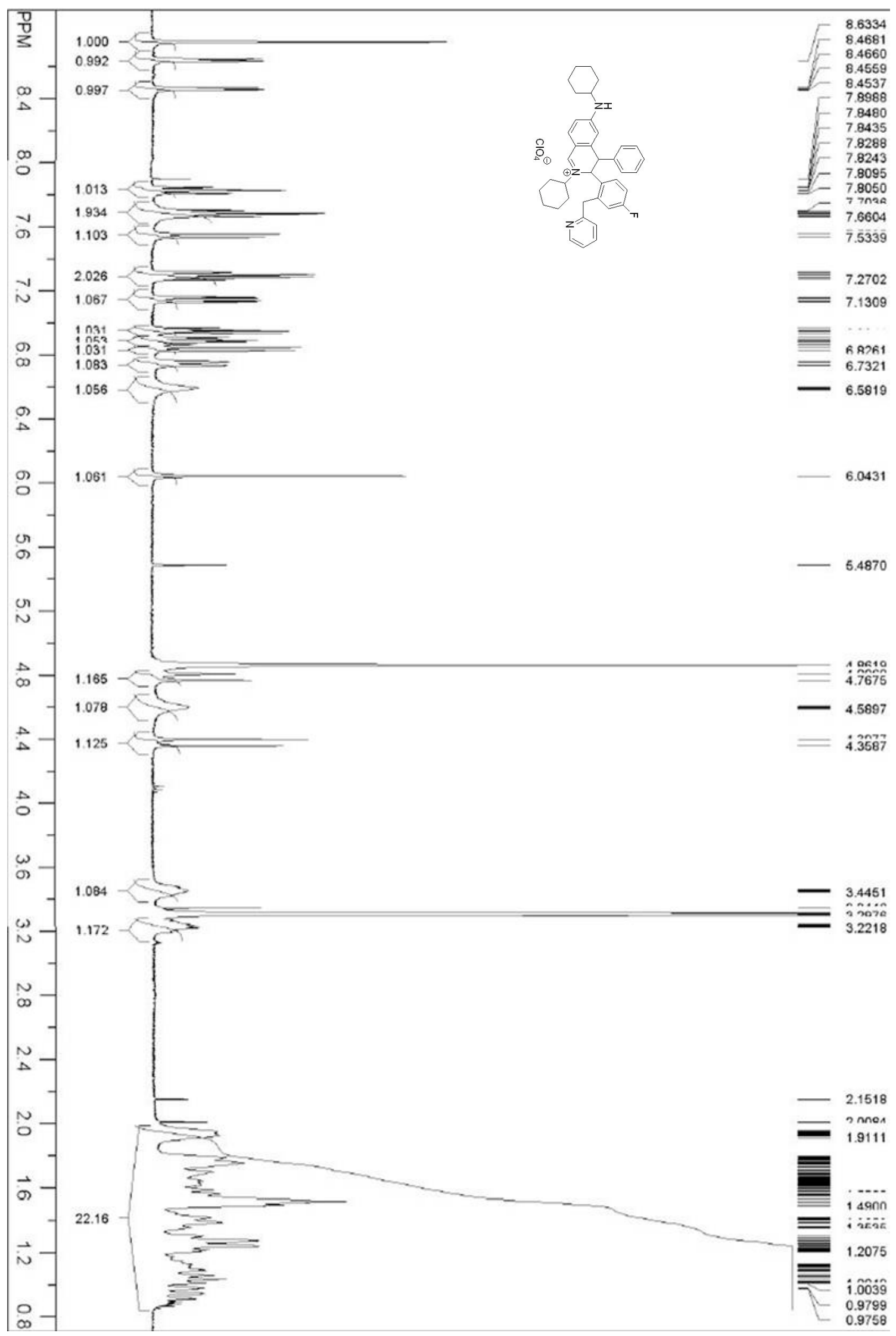




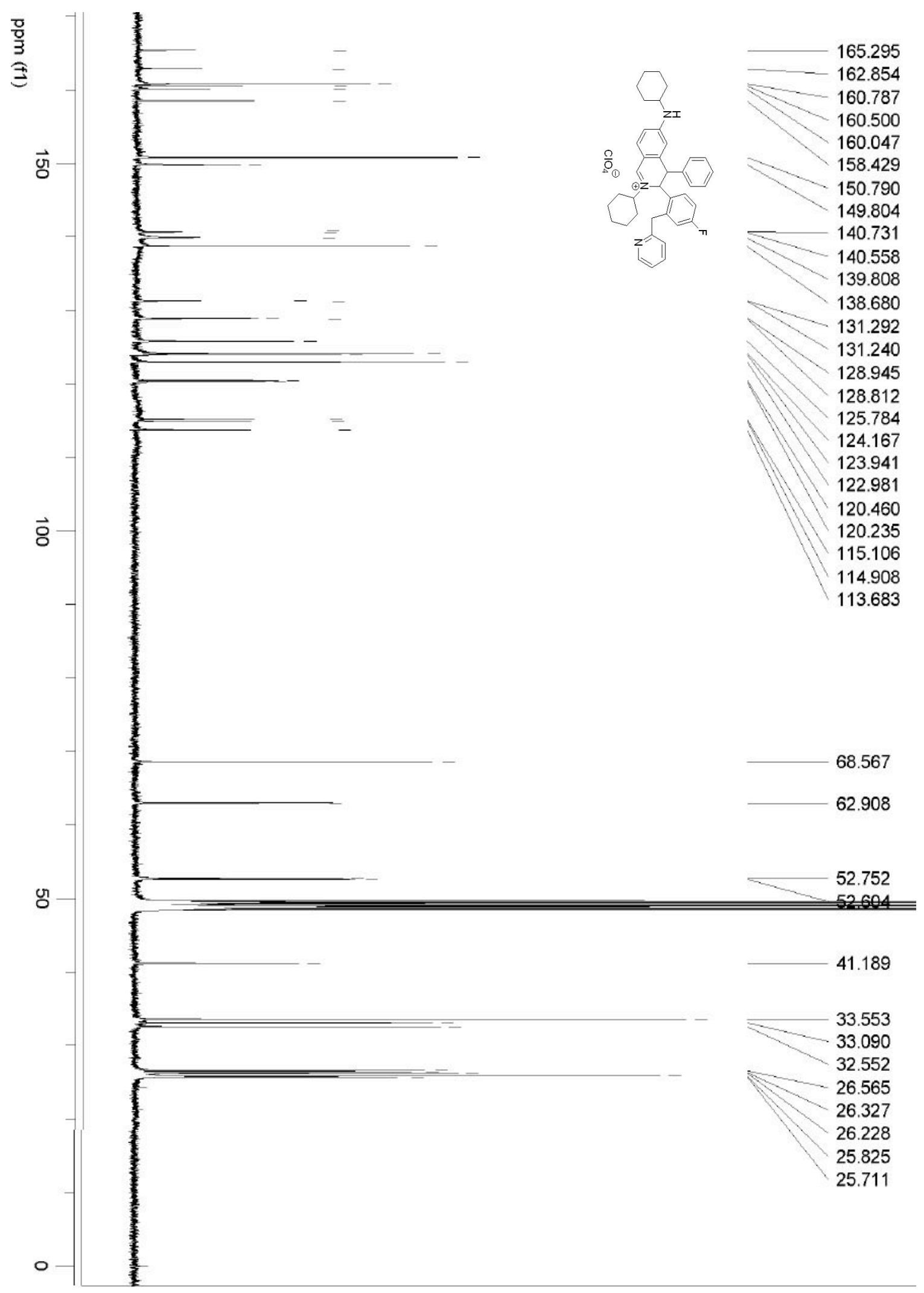




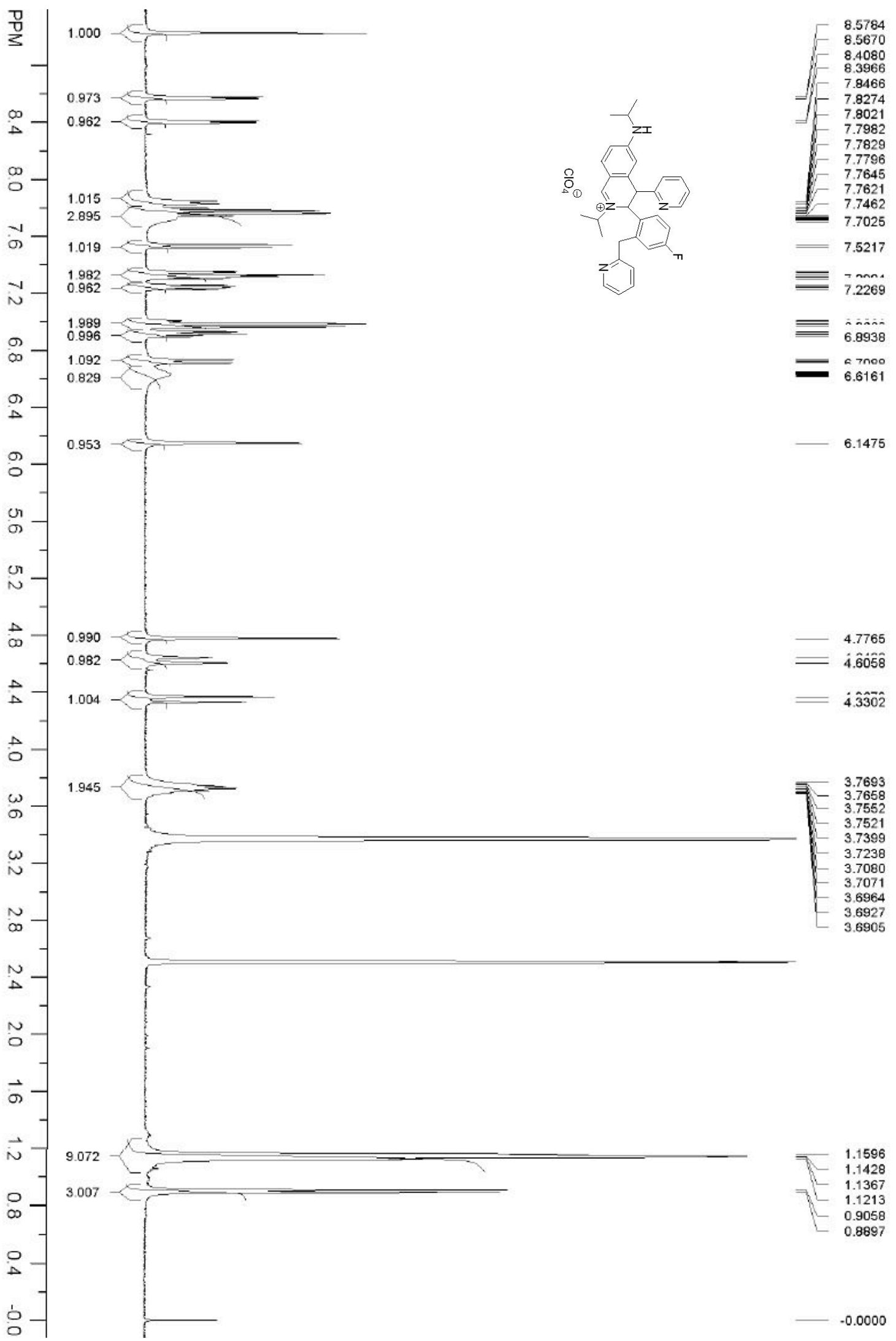




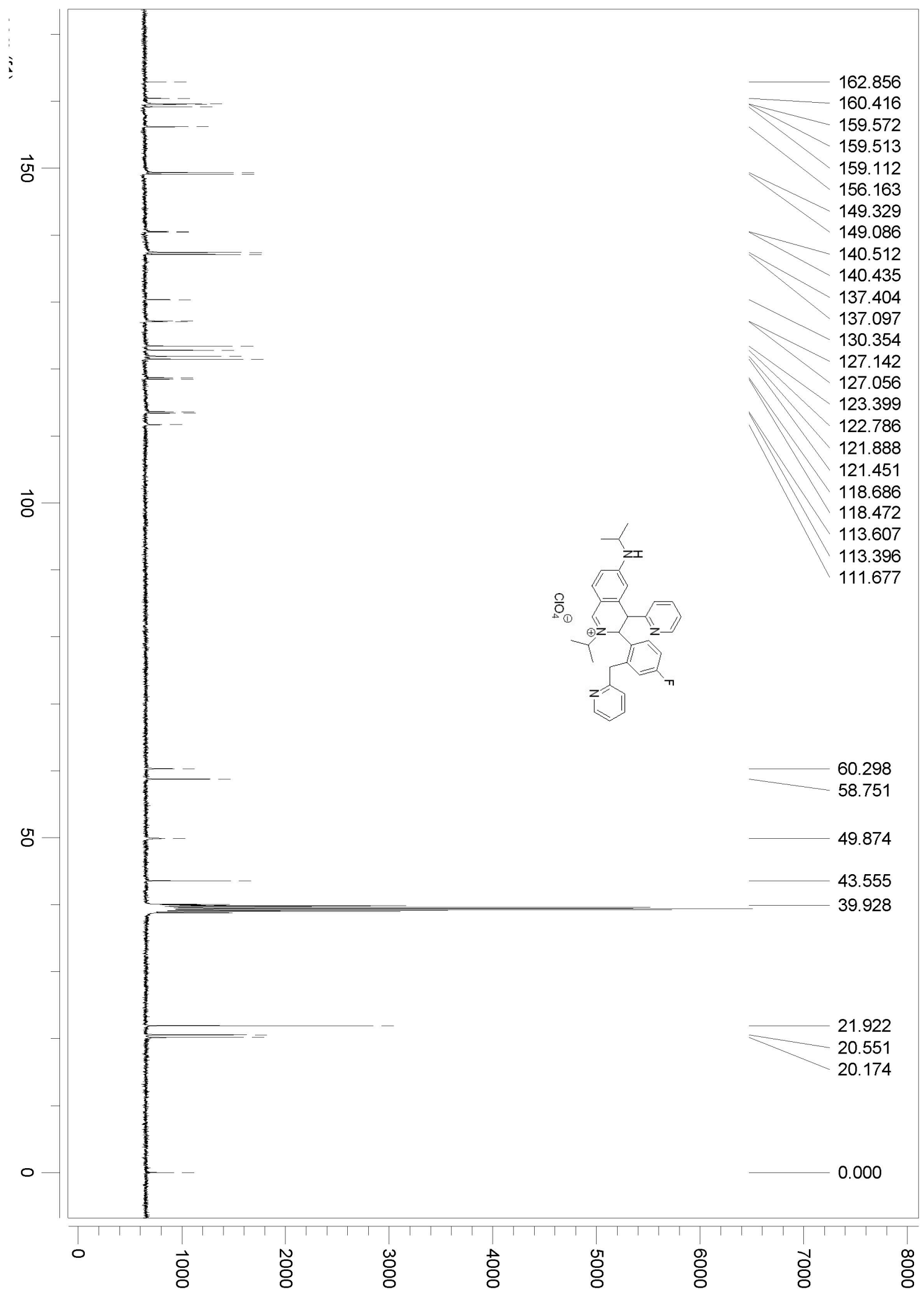




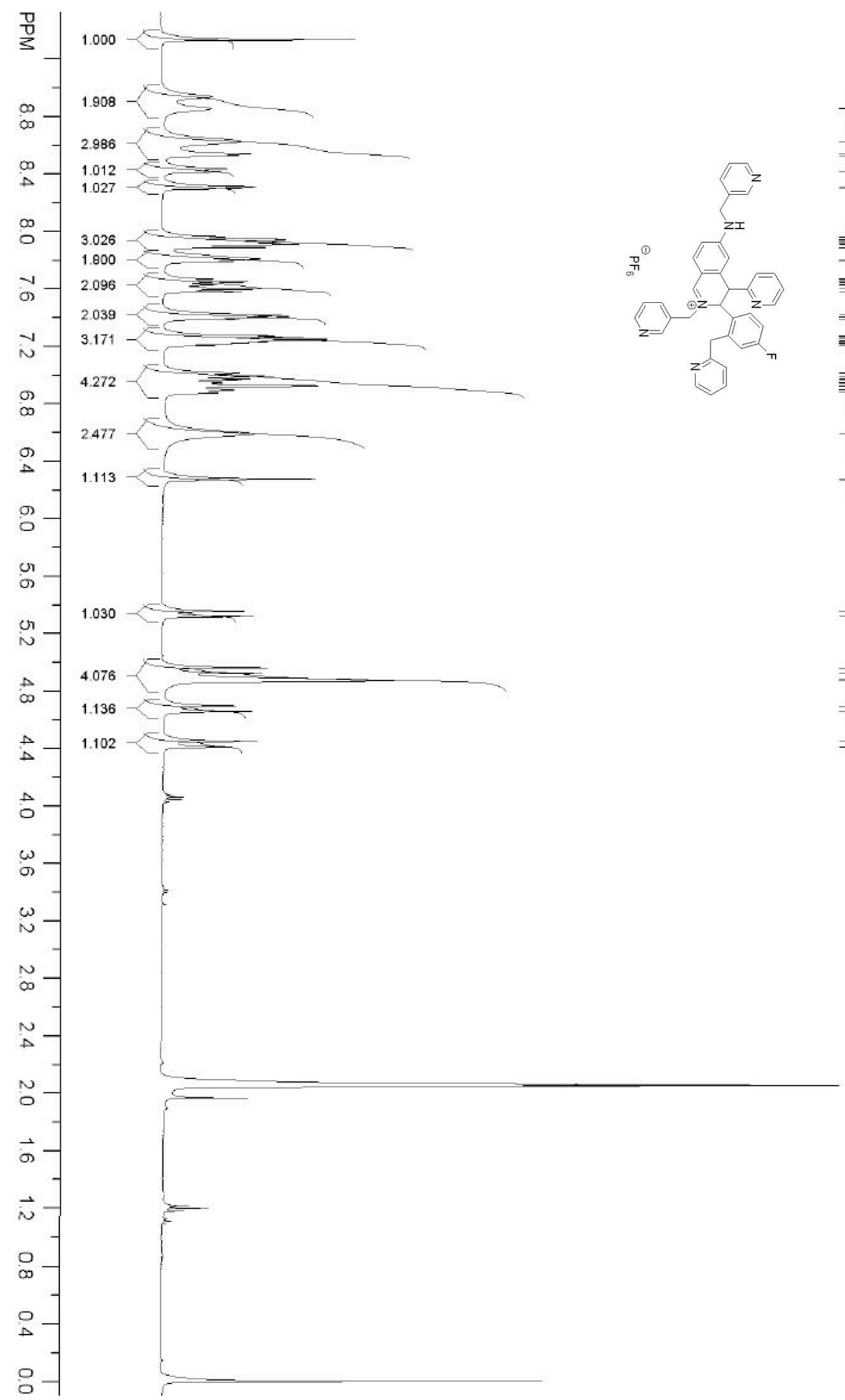




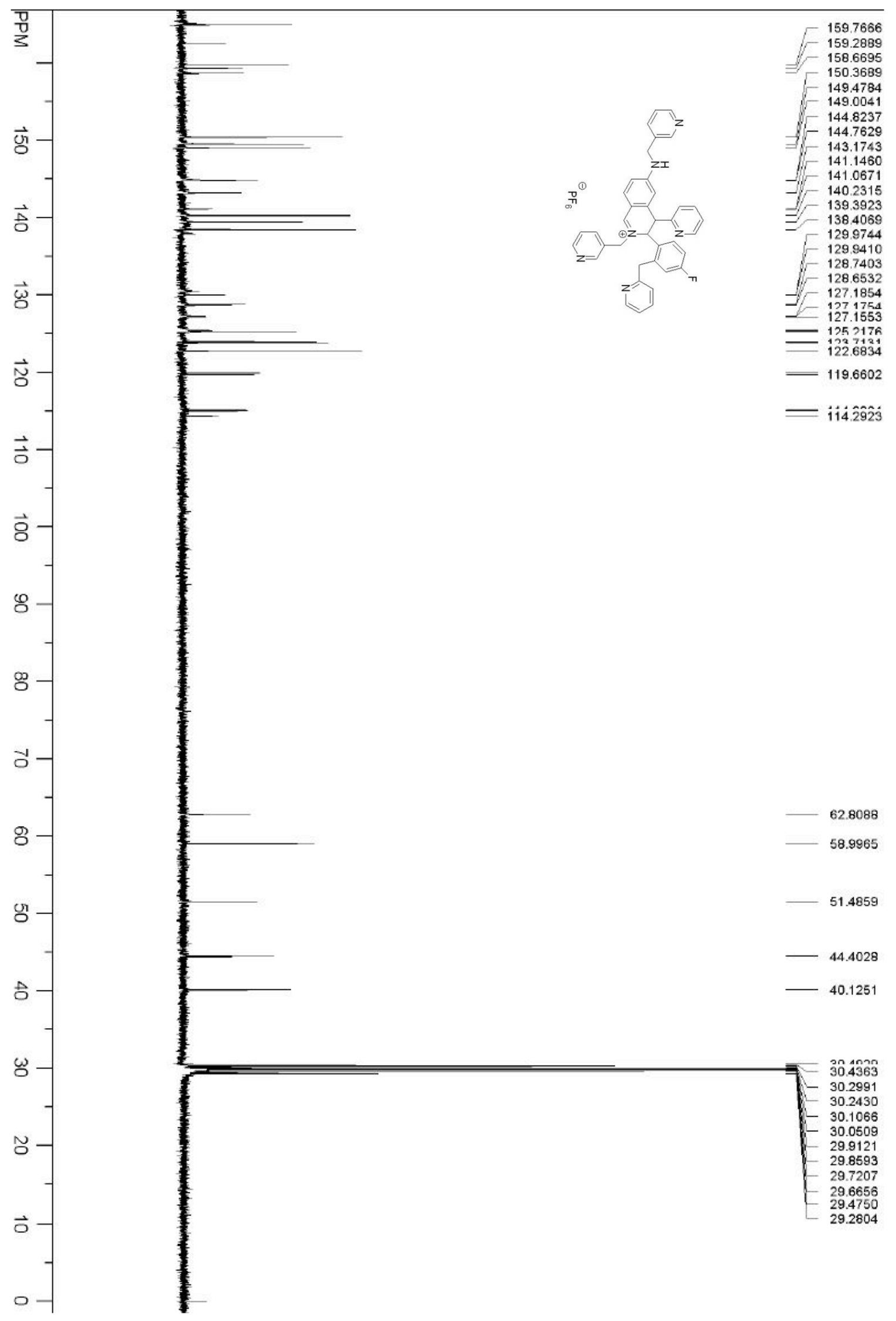




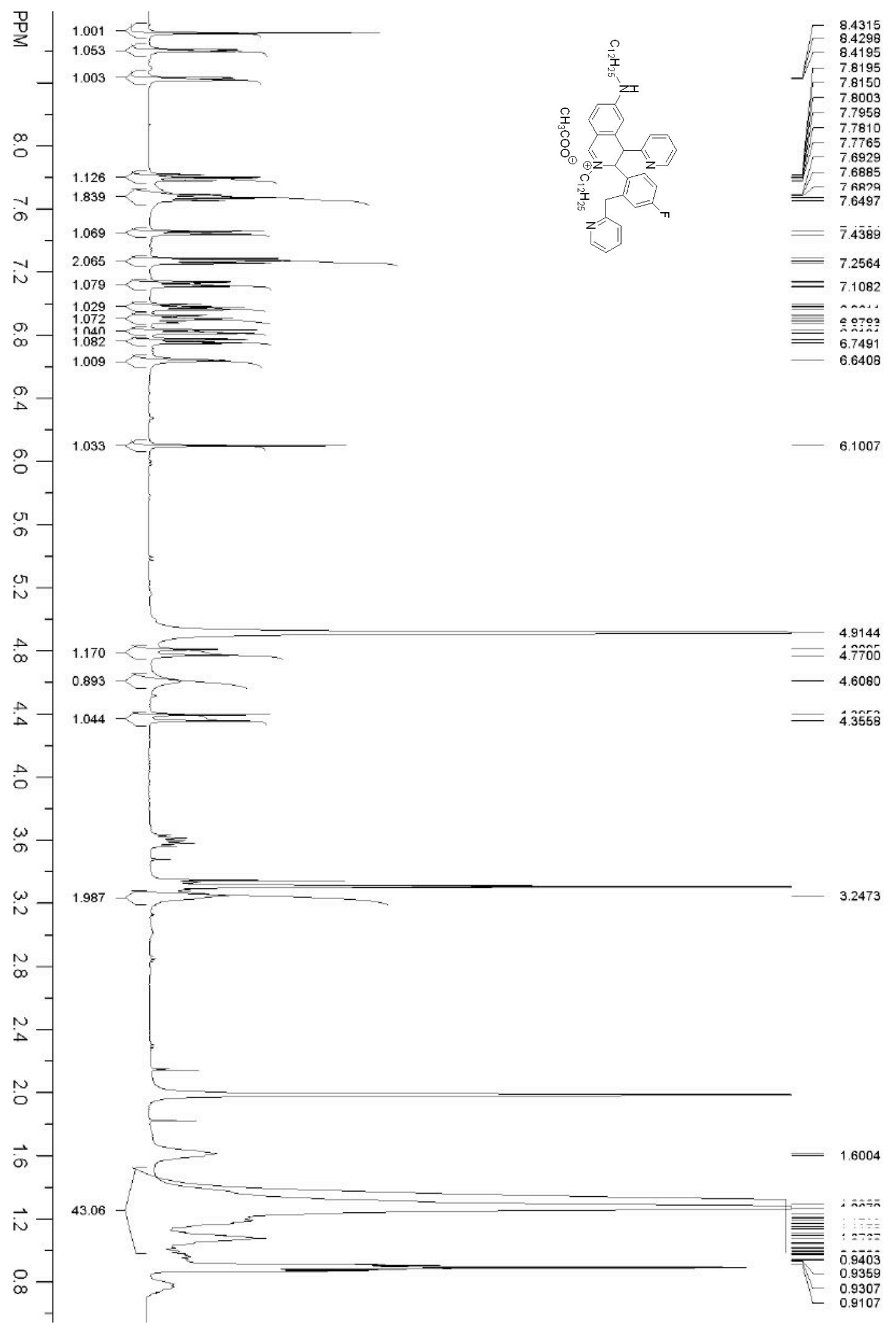




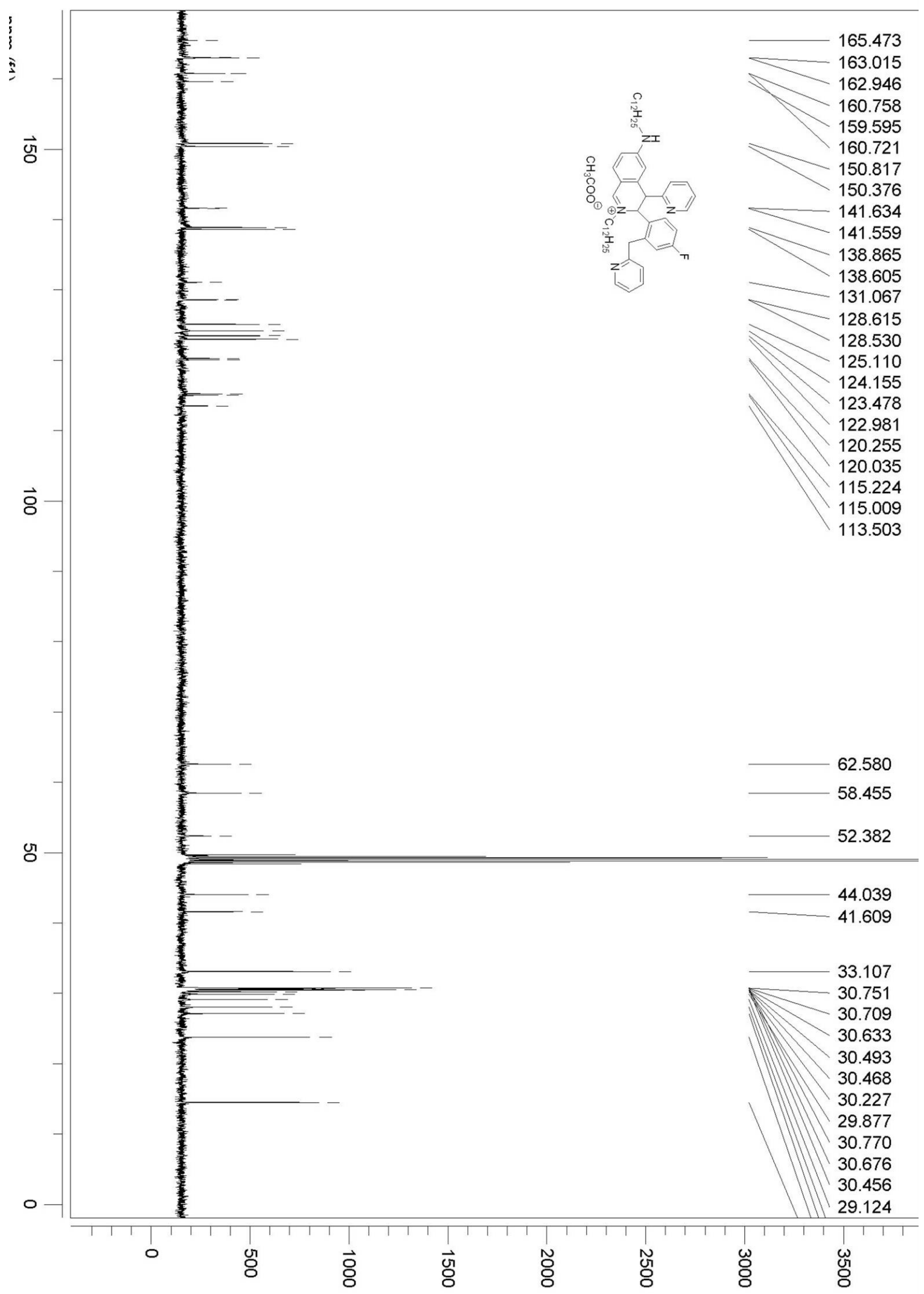




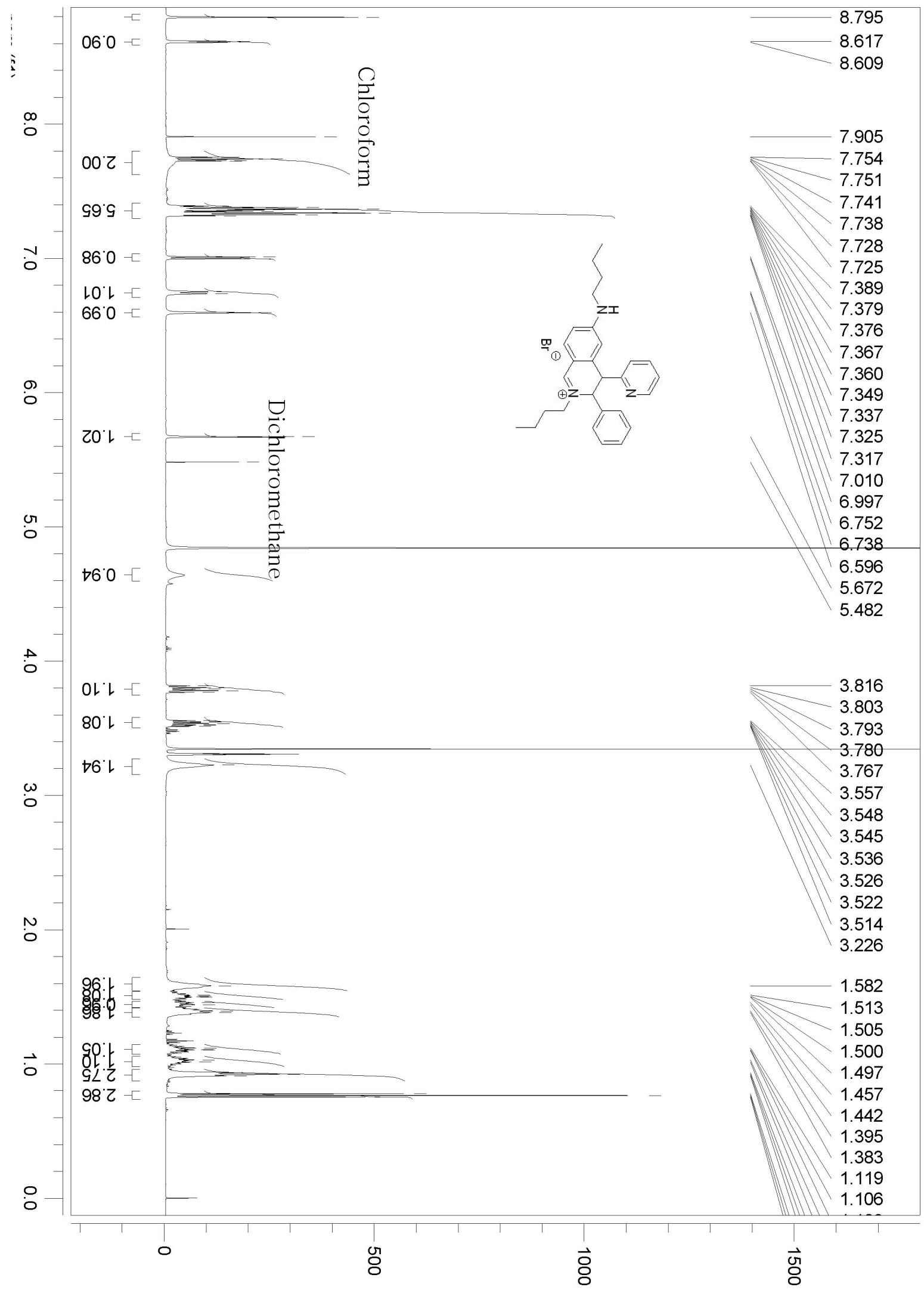




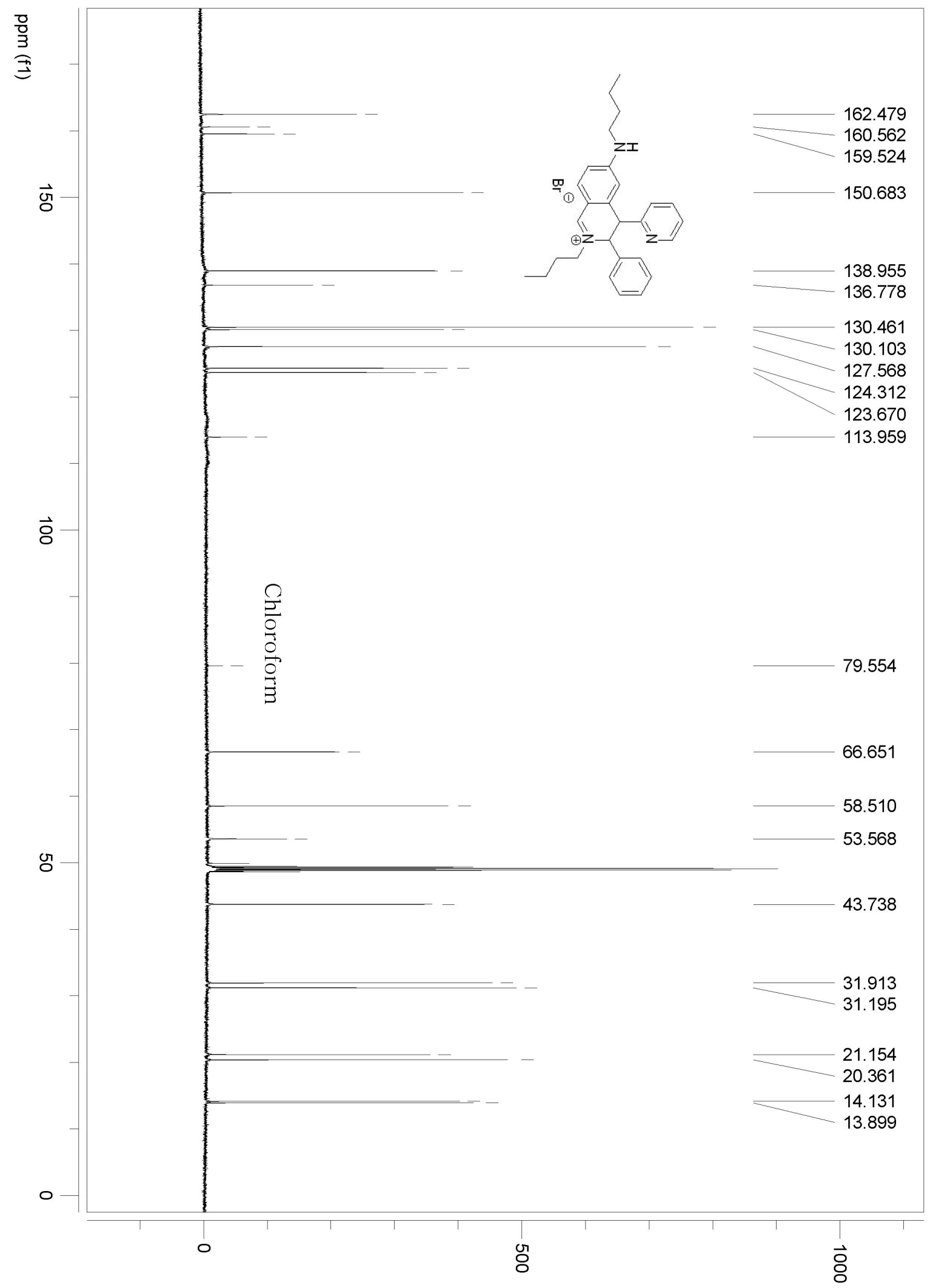




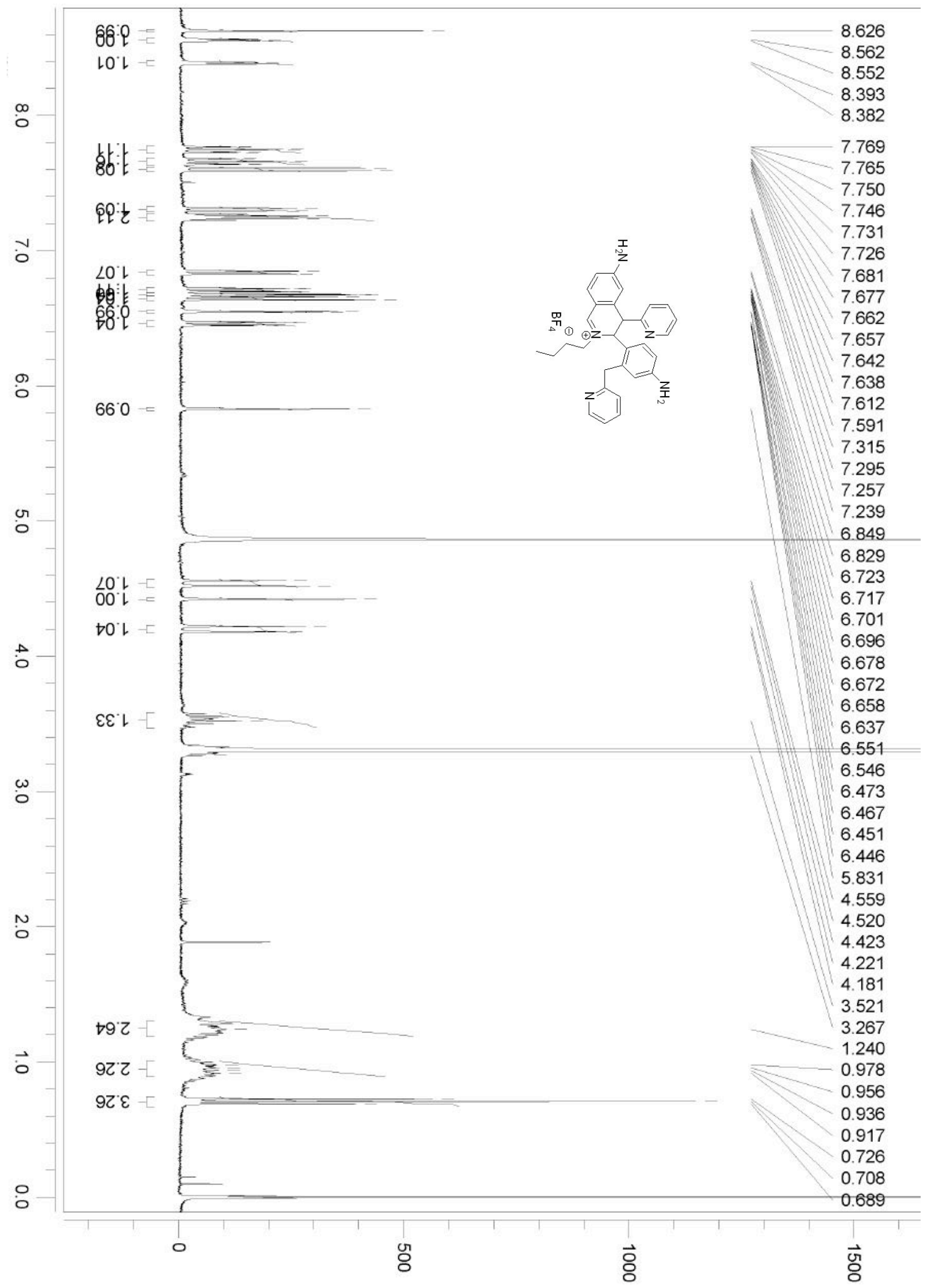




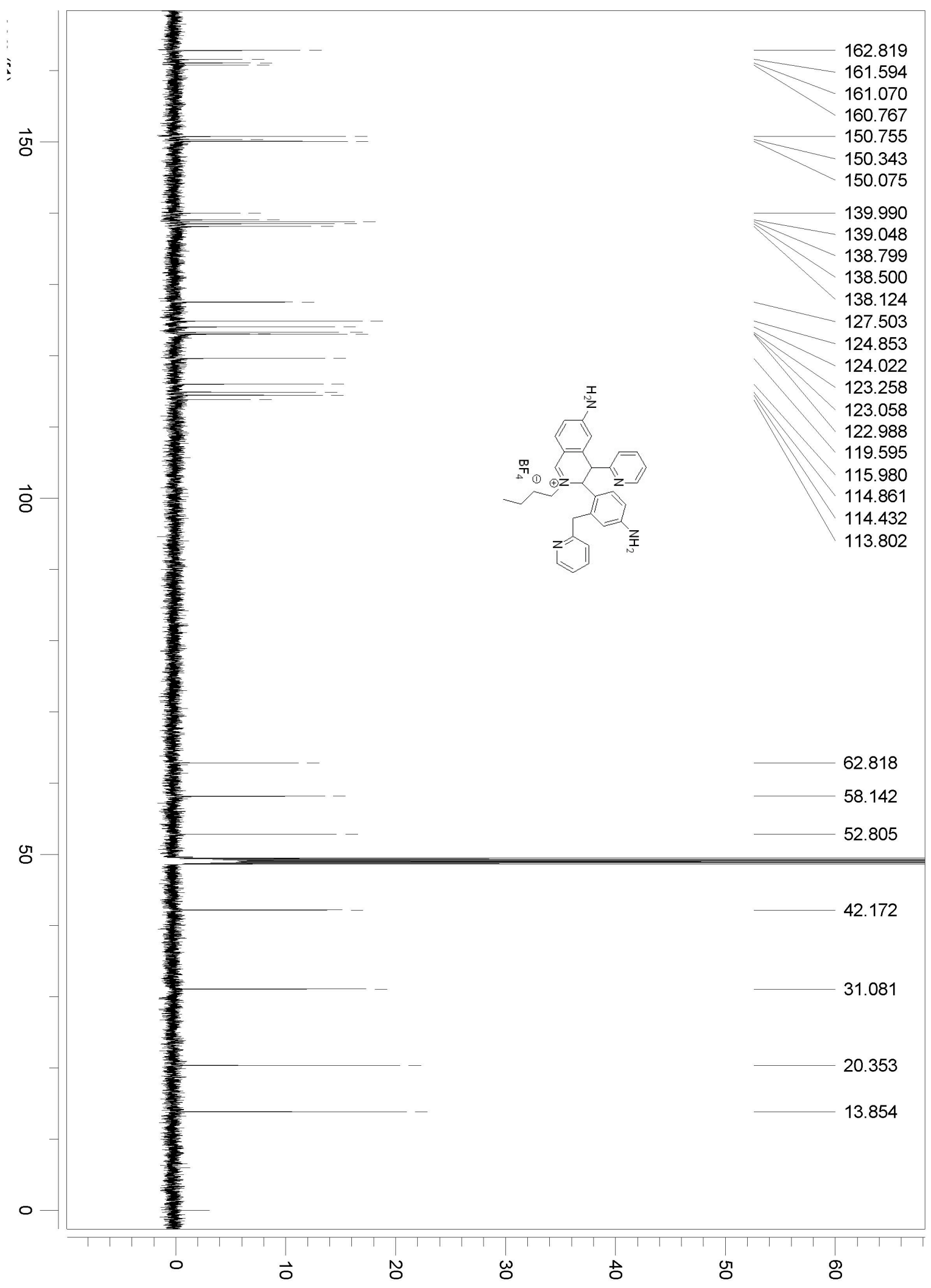




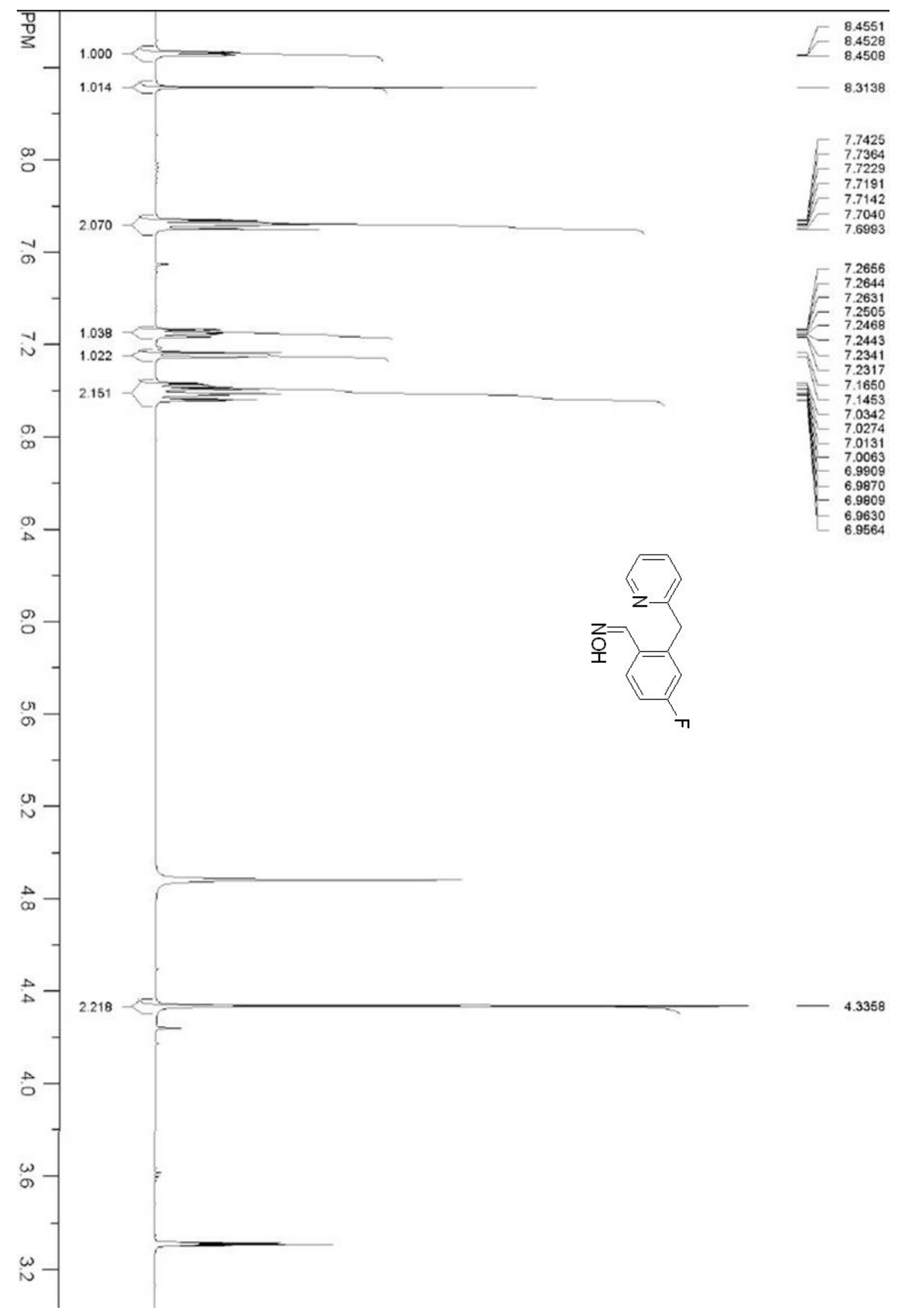




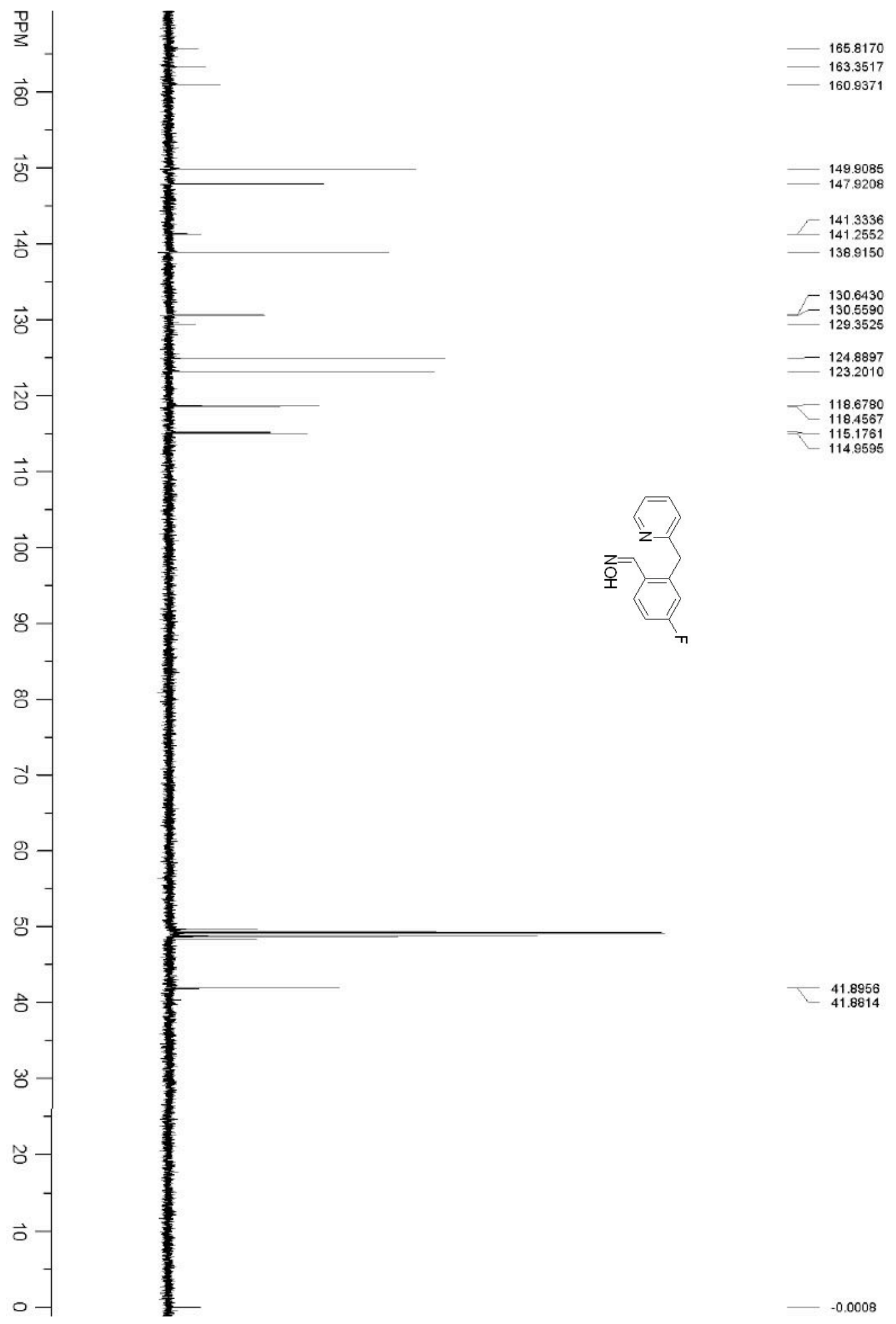

